\title{
Coronavirus transmissible gastroenteritis virus antagonizes the antiviral effect of the microRNA miR-27b via the IRE1 pathway
}

\author{
Changlin Wang ${ }^{2,3 \dagger}$, Mei Xue ${ }^{1 \dagger}$, Peng Wu ${ }^{2,3 \dagger}$, Honglei Wang ${ }^{2,3}$, Zhongqing Liu ${ }^{2,3}$, \\ Guangzheng $\mathrm{Wu}^{2,3}$, Pinghuang Liu ${ }^{1}$, Keliang Wang ${ }^{2,3^{*}}$, Wanhai $\mathrm{Xu}^{2,3 *} \& \mathrm{Li}^{*}$ Feng ${ }^{1^{*}}$ \\ ${ }^{1}$ State Key Laboratory of Veterinary Biotechnology, Harbin Veterinary Research Institute, Chinese Academy of Agricultural Sciences, \\ Harbin 150069, China; \\ ${ }^{2}$ Department of Urology, the Fourth Affiliated Hospital of Harbin Medical University, Harbin 150001, China; \\ ${ }^{3}$ NHC Key Laboratory of Molecular Probes and Targeted Diagnosis and Therapy, Harbin Medical University, Harbin 150001, China
}

Received May 1, 2021; accepted June 18, 2021; published online November 19, 2021

\begin{abstract}
Although the functional parameters of microRNAs (miRNAs) have been explored to some extent, the roles of these molecules in coronavirus infection and the regulatory mechanism of miRNAs in virus infection are still unclear. Transmissible gastroenteritis virus (TGEV) is an enteropathgenic coronavirus and causes high morbidity and mortality in suckling piglets. Here, we demonstrated that microRNA-27b-3p (miR-27b-3p) suppressed TGEV replication by directly targeting porcine suppressor of cytokine signaling 6 (SOCS6), while TGEV infection downregulated miR-27b-3p expression in swine testicular (ST) cells and in piglets. Mechanistically, the decrease of miR-27b-3p expression during TGEV infection was mediated by the activated inositolrequiring enzyme 1 (IRE1) pathway of the endoplasmic reticulum (ER) stress. Further studies showed that when ER stress was induced by TGEV, IRE1 acted as an RNase activated by autophosphorylation and unconventionally spliced mRNA encoding a potent transcription factor, X-box-binding protein 1 (Xbp1s). Xbp1s inhibited the transcription of miR-27 and ultimately reduced the production of miR-27b-3p. Therefore, our findings indicate that TGEV inhibits the expression of an anti-coronavirus microRNA through the IRE1 pathway and suggest a novel way in which coronavirus regulates the host cell response to infection.
\end{abstract}

coronavirus, transmissible gastroenteritis coronavirus (TGEV), microRNA, inositol-requiring enzyme 1 (IRE1), immune evasion

Citation: Wang, C., Xue, M., Wu, P., Wang, H., Liu, Z., Wu, G., Liu, P., Wang, K., Xu, W., and Feng, L. (2022). Coronavirus transmissible gastroenteritis virus antagonizes the antiviral effect of the microRNA miR-27b via the IRE1 pathway. Sci China Life Sci 65, 1413-1429. https://doi.org/10.1007/s11427-021$1967-\mathrm{x}$

\section{INTRODUCTION}

Coronaviruses are a family of enveloped viruses with a positive-sense, single-stranded RNA genome (Fung and Liu, 2014). Coronaviruses cause a variety of diseases in mammals and birds, ranging from enteritis in pigs and cows and upper respiratory diseases in chickens to potentially lethal respiratory infections in humans (Fehr and Perlman, 2015).

$\dagger$ Contributed equally to this work

*Corresponding authors (Keliang Wang, email: 25072641@qq.com; Wanhai Xu, email: xuwanhai@hrbmu.edu.cn; Li Feng, email: fengli_h@163.com)
Transmissible gastroenteritis virus (TGEV) is an intestinal coronavirus that can cause acute and highly contagious intestinal infectious diseases in pigs (Doyle and Hutchings, 1946; Pritchard, 1987).

The replication of coronaviruses is closely associated with cellular membrane compartments, especially the endoplasmic reticulum (ER) (Fung and Liu, 2014). Coronavirus infection of cultured cells has previously been shown to cause ER stress and induce the unfolded protein response (UPR), a process that restores the homeostasis of the intracellular environment and maintains cell survival 
(Fung et al., 2014). Three proteins located on the ER membrane are critical for monitoring ER stress and initiating the UPR: inositol-requiring enzyme 1 (IRE1), RNA-dependent protein kinase endoplasmic reticulum kinase (PERK) and activator of transcription 6 (ATF6) (Fung and Liu, 2014).

IRE1 is an ancient highly conserved ER stress response pathway that exists in all eukaryotic cells. ER stress can lead to the oligomerization and autophosphorylation of IRE1 to activate its RNA endonuclease activity. IRE1 can cleave Xbox-binding protein 1 (Xbp1) mRNA to its mature form (Xbp1 split or Xbp1s) (Calfon et al., 2002). Mature Xbp1 mRNA encodes a basic transcription factor containing a leucine zinc finger structure that can enhance the transcriptional activity of the molecular chaperone protein GRP78 (Lee et al., 2003). The up regulation of chaperone protein expression can promote the recovery of endoplasmic reticulum function, which is conducive to the correct folding and packaging of new proteins (Calfon et al., 2002).

MicroRNAs (miRNAs) are small noncoding RNAs composed of 17-25 nucleotides, which can regulate gene expression at the posttranscriptional level. The two key enzymes required for the maturation of these small regulators are the RNase III enzymes Drosha and Dicer, which cleave miRNA precursors and eventually lead to the formation of a mature miRNA duplex. MiRNAs mediate mRNA decay or translation inhibition by pairing with the complementary sequence of the $3^{\prime}$ untranslated region (UTR) of the target gene (Bartel, 2004; van Rooij and Olson, 2007). There is increasing evidence that viruses and cell-derived miRNAs play important roles in viral infection and immune regulation (Forster et al., 2015; O'Neill et al., 2011). For example, the human microRNA hsa-miR-125a-5p interferes with the expression of hepatitis $\mathrm{B}$ virus surface antigen to control viral replication (Potenza et al., 2011). SARS-CoV infection caused a large number of changes in miRNA expression, indicating that miRNAs are important components in regulating the host-virus infection response (Peng et al., 2011). miR-223 and miR-98 can interfere with the expression of ACE2, a key protein receptor in the host invasion of SARS-CoV, effectively explaining the successful replication and transmission of the virus in vivo (Mallick et al., 2009). The miR-30 family is a widely studied group of host miRNAs that play important roles in viral infection by regulating IFN signals (Ma et al., 2018). miR-27b attenuates TGEVinduced apoptosis by targeting RUNX1, suggesting that TGEV may function with miR-27b to regulate apoptosis in swine testicular (ST) cells (Zhao et al., 2016).

Although the current research mainly focuses on the identification of downstream targets of miRNAs, the upstream mechanism of miRNA regulation remains largely unclear. Recent publications have shown that activated IRE1 affects cell fate by directly degrading a subset of host miRNAs (miR-17, miR-30a, miR-34a, miR-96 and miR-
$125 \mathrm{~b}$ ) under persistent ER stress induced by chemicals or noninfectious diseases (Zhang et al., 2020). However, whether and how coronaviruses exploit IRE1 to manipulate miRNA expression for optimal viral infection remains unknown.

In this study, we reported an important biological function of the miR-27b-3p in coronavirus TGEV infection. We further found that when TGEV-mediated ER stress occurs, IRE1 acts as an RNase upon activation through autophosphorylation and unconventionally splices the mRNA encoding a potent transcription factor, Xbp1, while $\mathrm{Xbp} 1$ as a transcription factor inhibits the transcription of miR-27 and ultimately inhibits the production of miR-27b-3p, thus evades the antiviral effect of microRNA. This study revealed a new mechanism of coronavirus escaping from the host antiviral immune response.

\section{RESULTS}

\section{miR-27b suppresses TGEV propagation}

miR-27b is a highly conserved microRNA in vertebrates and has been demonstrated to play vital roles in cancers and viral infection (Ding et al., 2017). To investigate the role of miR27b-3p in TGEV propagation, we monitored TGEV infection in ST cells after transfecting different concentrations of miR$27 \mathrm{~b}-3 \mathrm{p}$ mimics $24 \mathrm{~h}$ prior to infection. Compared with the negative control (NC) mimics, the 80 and $160 \mathrm{nmol} \mathrm{L}^{-1} \mathrm{miR}$ $27 b-3 p$ mimics decreased the TGEV genome quantity and progeny viral titer by as much as 18.5 -fold (Figure $1 \mathrm{~A}$ and B). The suppression of TGEV infection by miR-27b-3p was further confirmed by Western blotting analysis and immunofluorescence assays (IFAs) using the antibodies against TGEV nucleocapsid protein (Figure 1C and D). Furthermore, the specific suppression of endogenous miR-27b-3p in ST cells by the miR-27b-3p inhibitor boosted TGEV infection compared with the NC inhibitor (NC-i) (Figure 1C-E). These results demonstrate that miR-27b-3p suppresses TGEV infection.

\section{miR-27b directly targets SOCS6}

To elucidate the underlying mechanisms of miR-27b-3p inhibiting the proliferation of TGEV, we performed computational analysis by using the TargetScan prediction program to identify the potential target genes of miR-27b-3p. Computational analysis indicates that miR-27b-3p may target SOCS6 through a conserved 3'UTR site in mammals (Figure $2 \mathrm{~A})$. Next, to explore whether miR-27b-3p can directly target SOCS6, the predicted target sites of SOCS6 3'UTR were cloned into a firefly luciferase reporter vector. In addition, a mutant vector was constructed to eliminate the possible recognition by replacing seven seed nucleotides (in which the 
A
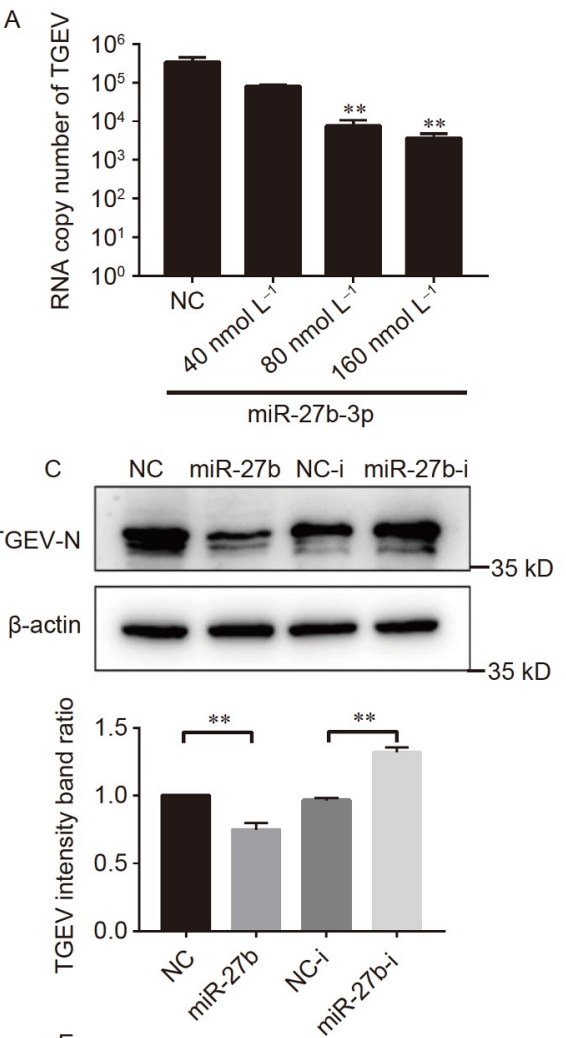

E

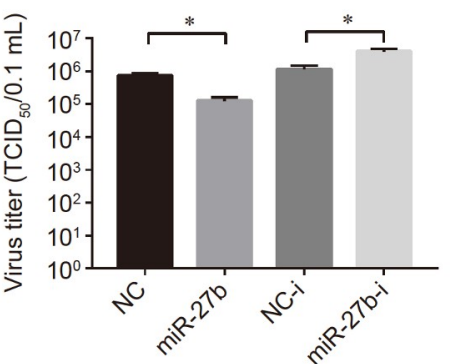

B
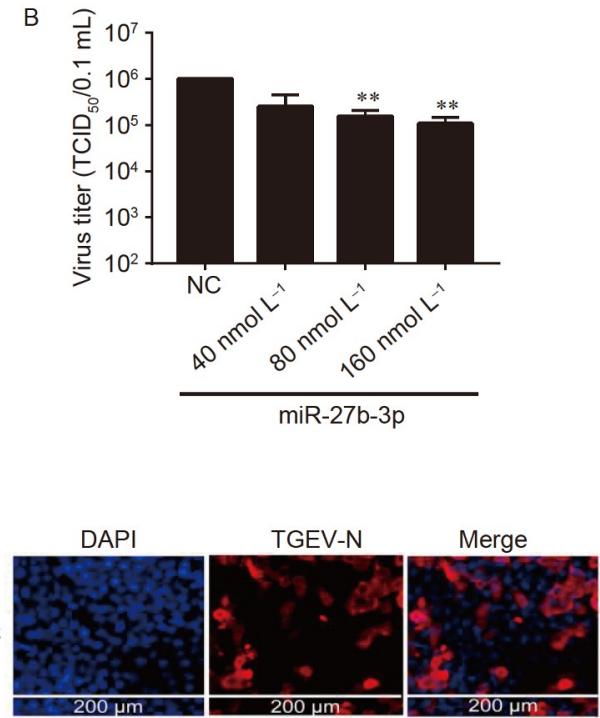

miR-27b
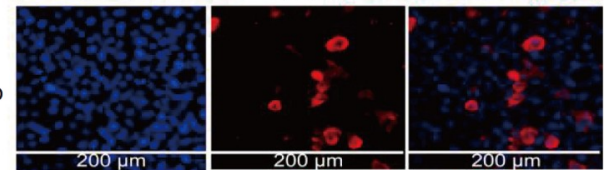

$\mathrm{NC}-\mathrm{i}$
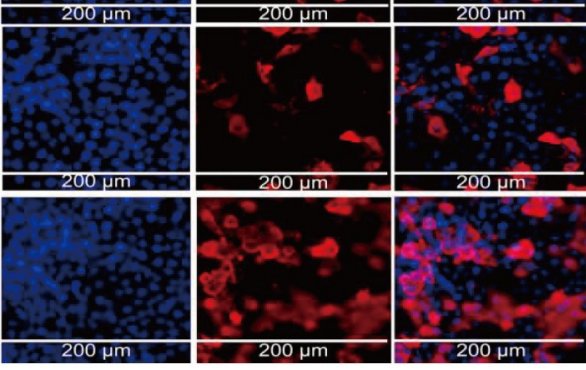

Figure 1 (Color online) miR-27b-3p inhibits the replication of TGEV. A and B, miR-27b-3p overexpression inhibited TGEV infection. ST cells were transfected with miR-27b-3p mimics at the indicated doses $\left(40,80\right.$ and $\left.160 \mathrm{nmol} \mathrm{L}{ }^{-1}\right)$ for $24 \mathrm{~h}$, followed by infection with TGEV for $24 \mathrm{~h}$ at a MOI of 0.1 . TGEV infection was determined at 24 hpi by RT-qPCR (A) or titration (B). C, The inhibitory effect of miR-27b-3p on TGEV N protein was detected by Western blot. D, The suppression of TGEV infection by miR-27b-3p was confirmed by IFA. E, ST cells were transfected with NC, $160 \mathrm{nmol} \mathrm{\textrm {L } ^ { - 1 }}$ miR-27b-3p mimics, NC inhibitor and miR-27b-3p mimics inhibitor for $24 \mathrm{~h}$, followed by infected with TGEV (MOI=1). TGEV titer was measured at 24 hpi.

target nucleotides in the sequence ACUGUGA were changed, resulting in the sequence CAGAGUG) (Figure 2A). Compared with the $\mathrm{NC}$ mimic treatment, overexpression of miR-27b-3p in ST cells reduced the luciferase reporter activity containing SOCS6 wild-type target sequence, but did not reduce the luciferase reporter activity containing SOCS6 mutant target site. In contrast, compared with the NC inhibitor, the miR-27b-3p inhibitor increased the activity of the luciferase reporter containing the SOCS6 wild-type target sequence but not that of the luciferase reporter containing the mutant target site (Figure 2B). To further confirm that SOCS6 is a direct target of miR-27b-3p, we detected the transcript and protein level of SOCS6 in ST cells transfected with the miR-27b-3p mimics or inhibitor. As expected, the miR-27b-3p mimics significantly reduced the transcription level of SOCS6 in ST cells (Figure 2C). The diminished expression of SOCS6 induced by miR-27b-3p overexpression was verified by Western blotting (Figure 2D). In contrast, miR-27b-3p inhibitor increased SOCS6 expression in ST cells compared with $\mathrm{NC}$ inhibitor (Figure 2C and D). Regulation of miR-27b-3p abundance affects SOCS6 expression in ST cells infected with TGEV, similar to that in TGEV-uninfected ST cells (Figure 2D). Altogether, these data demonstrate that miR-27b-3p down-regulates the expression of SOCS6 by directly targeting its $3^{\prime} \mathrm{UTR}$.

SOCS family members are effective endogenous negative regulators of JAK-STAT signal transduction. To investigate the regulation of SOCS6 by TGEV infection, we examined the expression of SOCS6. TGEV infection substantially elevated the expression of SOCS6 in ST cells and exhibited a 


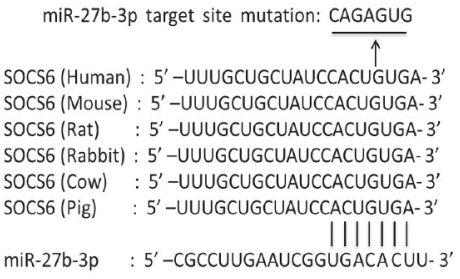

B

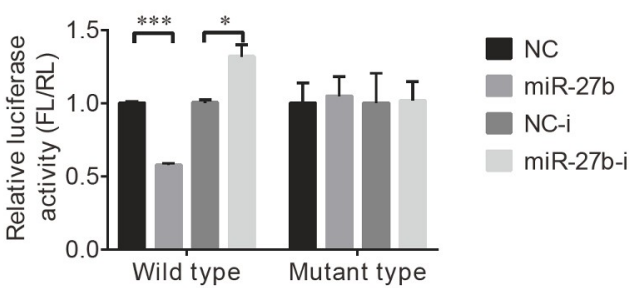

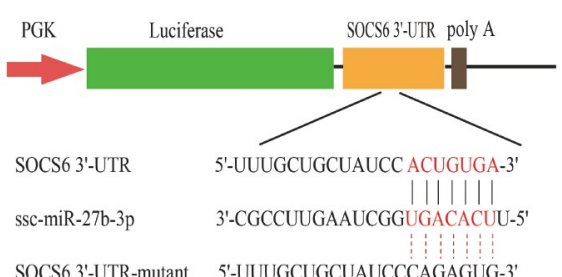

C
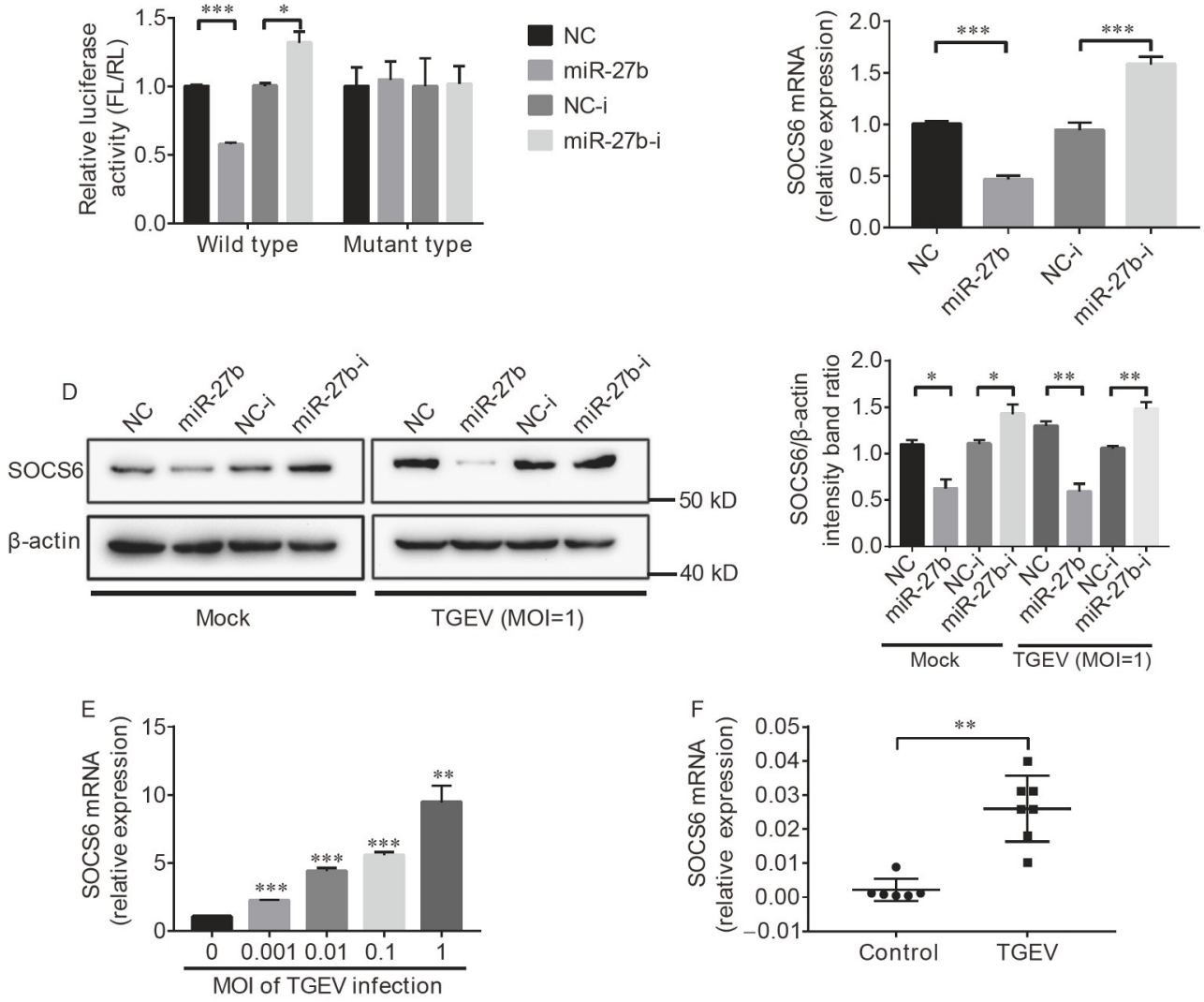

Figure 2 (Color online) miR-27b negatively regulates the expression of porcine SOCS6. A, Diagram of the predicted miR-27b binding sites in SOCS6 3' UTR of different species. The predicted target sites and mutated target sites of miR-27b-3p are underlined and were mutated as indicated. B, miR-27b directly targets SOCS6 3'UTR. ST cells were cotransfected with SOCS6 wild-type or mutant luciferase vectors $(500 \mathrm{ng})$ and $160 \mathrm{nmol} \mathrm{L}^{-1}$ miR-27b-3p mimics or NC mimics, miR-27b-3p inhibitor, or NC inhibitor. After 48 h, transfected cells were collected and lysed for dual-luciferase reporter assay. C, miR-27b decreases the levels of SOCS6 mRNA. ST cells were transfected with $160 \mathrm{nmol} \mathrm{L}{ }^{-1} \mathrm{NC}$ mimics, miR-27b-3p mimics, NC inhibitor, or miR-27b-3p inhibitor. The expression levels of SOCS6 were analyzed by RT-qPCR at $48 \mathrm{~h}$ after transfection. D, The suppression of SOCS6 protein levels by miR-27b-3p under TGEVuninfected and -infected conditions. At $48 \mathrm{~h}$ post-transfection as described in the legend to panel C, ST cells were lysed and subjected to Western blotting. The densitometric analysis was performed using ImageJ. E, TGEV infection up-regulated SOCS6 expression. The SOCS6 expression levels in ST cells were measured by RT-qPCR at 24 hpi at different MOIs. F, Elevated expression of SOCS6 in the ileum after TGEV infection. The expression of SOCS6 in the ileum at 48 hpi was quantified by RT-qPCR. Bars represent the means \pm SEM. $*, P<0.05 ; * *, P<0.01 ; * * *, P<0.001$.

dose-dependent induction of MOIs (Figure 2E). Consistent with the in vitro SOCS6 expression results, the expression of SOCS6 (Figure 2F) in the TGEV-infected ileum was also upregulated more than 15 -fold. Taken together, these results demonstrate that TGEV infection up-regulates the expression of SOCS6.

\section{TGEV infection down-regulates miR-27b-3p expression}

The aforementioned experiments demonstrated that the specific concentration range of miR-27b-3p had a significant impact on viral growth capacity. Although the manipulation of miR-27b-3p can potentially be exploited in a therapeutic context, we expect that the miRNAs most relevant to the infection process might be naturally up- or down-regulated upon infection, either as a host response to viral infection or as a viral strategy to manipulate the host. To determine whether miR-27b-3p can be regulated by TGEV infection, we monitored miR-27b-3p expression in ST cells after TGEV infection at different MOIs. TGEV infection significantly reduced the levels of miR-27b-3p 24 hpi compared with mock infection and displayed a MOI dose-dependent 
response (Figure 3A). Further, Northern blot was used to verify the change of miR-27b-3p, and the results showed that TGEV infection substantially reduced the expression of miR27b-3p in ST cells and exhibited a dose-dependent decrease of MOIs (Figure 3B). TGEV infection of ST cells was determined by virus titer (Figure 3C). To determine which stage the inhibition of miR-27b-3p by TGEV infection occurs at, we analyzed the expression of miR-27b-3p at different time points after TGEV infection. TGEV infection with a MOI resulted in typical cytopathic effects (CPEs), including cell fusion $24 \mathrm{hpi}$ and approximately $50 \%$ cell death 48 hpi. miR-27b-3p decreased after 12 hpi and then gradually decreased to $48 \mathrm{hpi}$ (Figure 3D). It is suggested that TGEV infection reduced the abundance of miR-27b-3p in the late stage of infection.

TGEV mainly infects villous epithelial cells of the small intestine in piglets, causing watery diarrhea. To evaluate whether TGEV infection can also reduce the expression of miR-27b-3p in piglets, we quantified the expression of miR$27 b-3 p$ in the ileum of piglets at $48 \mathrm{hpi}$. The abundance of miR-27b-3p in the ileum of the TGEV-infected group was 5.8-fold lower than that of the uninfected control group (Figure 3E). TGEV infection in the ileum was confirmed by quantifying the TGEV genome (Figure 3F). In addition, Northern blot was used to detect the expression of miR-27b$3 p$ in different intestinal tissues. The results showed that the miR-27b-3p was more abundant in ileum than in other intestinal tissues and the expression of miR-27b-3p was downregulated in TGEV infected tissues compared with normal tissues (Figure 3G). These results showed that TGEV infection reduced the expression of miR-27b-3p.

\section{TGEV-induced ER stress can down-regulate miR-27b expression}

In a previous study, we found that ER stress is closely related to the expression of microRNAs (Ma et al., 2018). To evaluate whether ER stress regulates the expression of miR-27b$3 p$, we first analyzed the expression level of miR-27b-3p in ST cells treated with thapsigargin ( $\mathrm{Tg})$, an ER stress inducer. We found that $\mathrm{Tg}$ treatment significantly reduced the abundance of miR-27b-3p in a dose-dependent manner (Figure 4A). The expression of miR-27b-3p was decreased $2 \mathrm{~h}$ after $\mathrm{Tg}$ treatment and then decreased to $48 \mathrm{~h}$ after treatment (Figure 4B), indicating that Tg-derived ER stress reduced miR-27b-3p abundance.

Next, we investigated the mechanisms critical for the inhibition of miR-27b-3p by TGEV infection. IRE1, a highly conserved ER stress sensor with protein kinase and RNase functions, can degrade not only mRNAs under ER stress but also miRNAs. Our previous studies confirmed that TGEV infection caused substantial cytoplasmic cleavage of the Xbplu transcript into the Xbp1s transcription factor starting at $24 \mathrm{hpi}$, indicating that IRE1 was activated by TGEV infection (Ma et al., 2018). And the activation of the pathway can be blocked by $4 \mu 8 \mathrm{c}$, a highly specific and selective inhibitor of IRE1 RNase activity.

To verify whether IRE1 pathway is involved in the regulation of miR-27b-3p, the miRNA expression levels of ST cells infected with TGEV or treated with $4 \mu 8 \mathrm{c}$ were detected by RNA sequencing (RNA-Seq). In the comparison of the cell group and TGEV group, 15 differentially expressed miRNAs were identified, including 1 up-regulated and 14 down-regulated miRNAs. In the DMSO group and the $4 \mu 8 \mathrm{c}$ group, 25 differentially expressed miRNAs were identified, including 3 up-regulated and 22 down-regulated miRNAs (Figure 4C). Then the fourteen down-regulated miRNAs of the cell group $v s$. TGEV group was compared with the three up-regulated miRNAs in the DMSO group $v s .4 \mu 8 \mathrm{c}$ group. The venn diagram showed that two groups shared only one microRNA, namely ssc-miR-27b-3p, which was downregulated after TGEV infection and up-regulated after $4 \mu 8 \mathrm{c}$ treatment (Figure 4D). Transcriptome analysis showed that the IRE1 pathway may be involved in the expression of miR27b-3p.

\section{IRE1 $\alpha$-mediated UPR induction suppresses miR-27b-3p expression}

To verify that activated IRE1 is critical for the down-regulation of miR-27b-3p, we monitored the expression of miR27b-3p in TGEV-infected or Tg-treated cells after inhibiting IRE1 function with $4 \mu 8 \mathrm{c}$. The inhibition of IRE1 RNase by $4 \mu 8 \mathrm{c}$ almost completely abolished the suppression of miR$27 b-3 p$ by Tg or TGEV (Figure $5 \mathrm{~A}$ and B). To verify the contribution of IRE1 to miR-27b-3p expression, we knocked down IRE1 expression by specific small interfering RNAs (siRNAs), and the efficiency of IRE1 knockdown was confirmed by Western blotting (Figure 5C). The silencing of IRE1 by siRNAs significantly reversed the decrease in miR27b-3p induced by TGEV infection (Figure 5D) or Tg treatment (Figure 5E) $(P<0.05)$. In addition, the efficiency of miR-27b-3p was restored by IRE1 siRNAs (siIRE1\#1 to siIRE1\#3) in a manner that correlated with the knockdown efficiency of IRE1 siRNAs (Figure 5C-E). Taken together, these results show that activated IRE1 reduces miR-27b-3p expression.

Considering that TGEV can also activate the PERK and ATF6 pathways, we sought to determine whether the PERK and ATF6 pathways are involved in the regulation of miR27b, and therefore, ST cells were treated with a PERK-specific inhibitor (GSK2606414) and then infected with TGEV. The results showed that miR-27b expression was not affected (Figure 5F). Further, PERK and ATF6 were knocked down by transfection with shPERK- or ATF6-specific siRNA duplexes. The efficiency of the PERK and ATF6 knockdown 


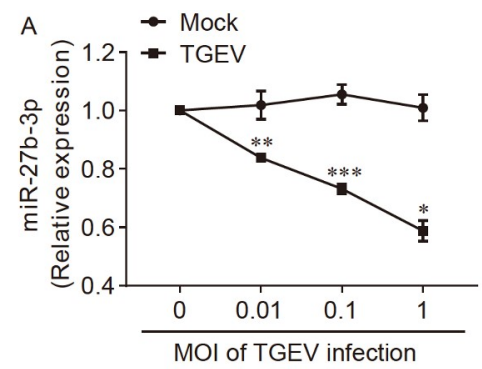

$\mathrm{C}$
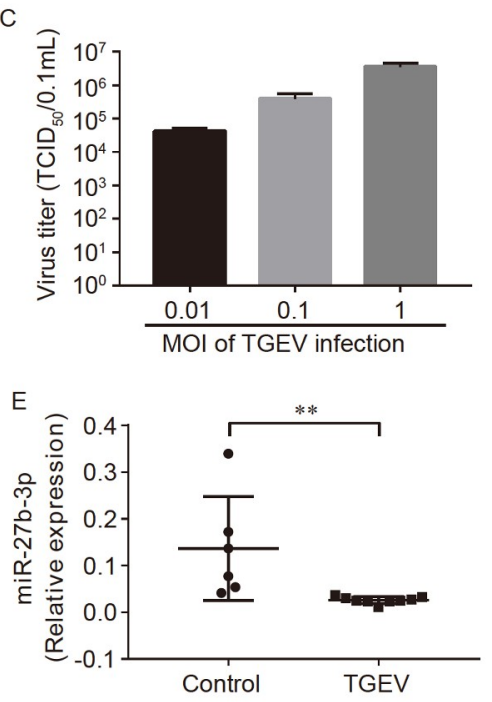

B

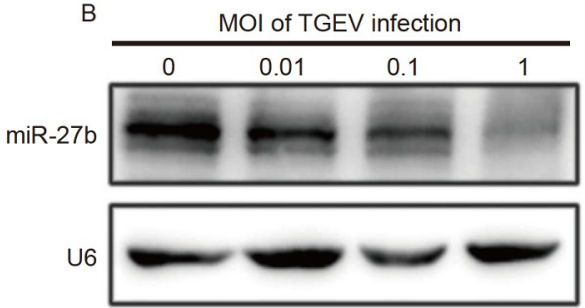

$\mathrm{D} \rightarrow$ Mock

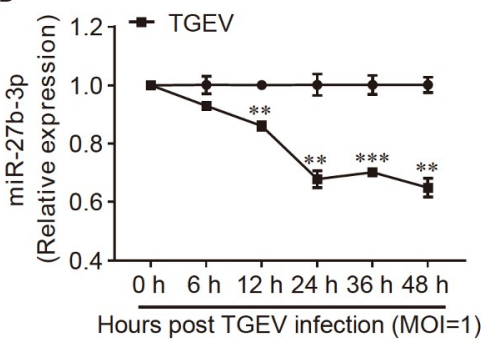

$\mathrm{F}$ 㟶

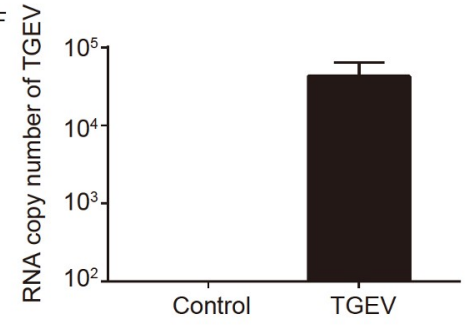

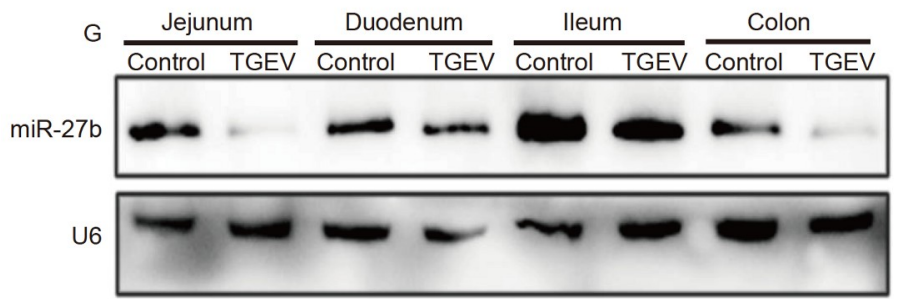

Figure 3 TGEV infection suppresses miR-27b-3p expression in ST cells and in piglets. A-C, TGEV infection downregulated miR-27b-3p expression in vitro. The miR-27b-3p levels in TGEV infected ST cells were measured by RT-qPCR (A) and Northern blot (B) at 24 hpi at different MOIs. TGEV infection was measured by RT-qPCR (C). D, For time kinetics, the levels of miR-27b-3p in ST cells were quantified at the indicated time points after infection with TGEV at a MOI of 0.1. The results from three independent experiments are shown. E and F, TGEV infection suppressed miR-27b-3p expression in piglets. Piglets were orally inoculated with $5 \mathrm{~mL} 1 \times 10^{5} \mathrm{TCID}_{50}$ TGEV or DMEM. Total cellular RNA from each ileum was collected at 48 hpi, and the levels of miR27b-3p (E) and TGEV viral RNA in the ileum (F) were measured by RT-qPCR. G, The suppression of miR-27b-3p in TGEV infected tissues was confirmed by Northern blot.

was confirmed by Western blotting (Figure 5G and I). The expression of miR-27b was not affected by knocking down PERK or ATF6 (Figure 5H and J), indicating that the PERK and ATF6 pathways are not involved in the regulation of miR-27b.

\section{Mechanism of TGEV inhibiting miR-27b-3p expression}

There are several processing steps from coding of primary transcripts to the formation of mature miRNAs. Primary miRNAs are cleaved to produce precursor miRNAs by a nuclear RNase III-type enzyme, Drosha, and its cofactor, DGCR8. Following nuclear processing, precursor miRNAs are exported from the nucleus into the cytoplasm by Ex- portin-5. These precursors are then cleaved by Dicer, and 22-bp miRNA duplexes are released. The resulting RNA duplex is loaded onto RISC, which contains Ago2. One strand (the guide strand) of the RNA duplex remains in RISC and functions as the mature miRNA (Kai and Pasquinelli, 2010). To investigate whether the changes in the miRNA expression levels are the result of the altered expression of key factors in the miRNA biogenesis pathway, we analyzed the mRNA expression levels of five miRNA processing factors, including DGCR8, Drosha, Exportin-5, Dicer, and Ago2, in ER-stressed ST cells $24 \mathrm{~h}$ after TGEV infection or $\mathrm{Tg}$ treatment. The results showed that there was no significant change in the expression of these five proteins (Figure 6A). Further, we perform nuclear run-on assay 

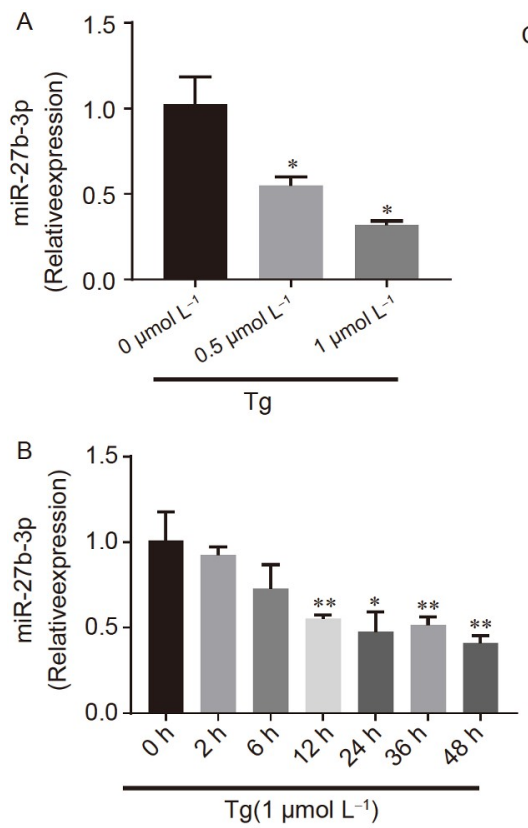

D

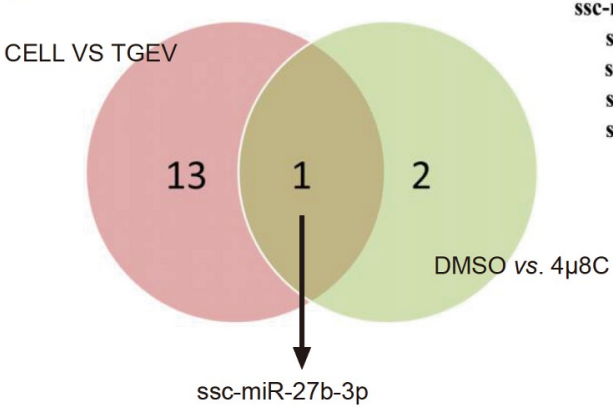

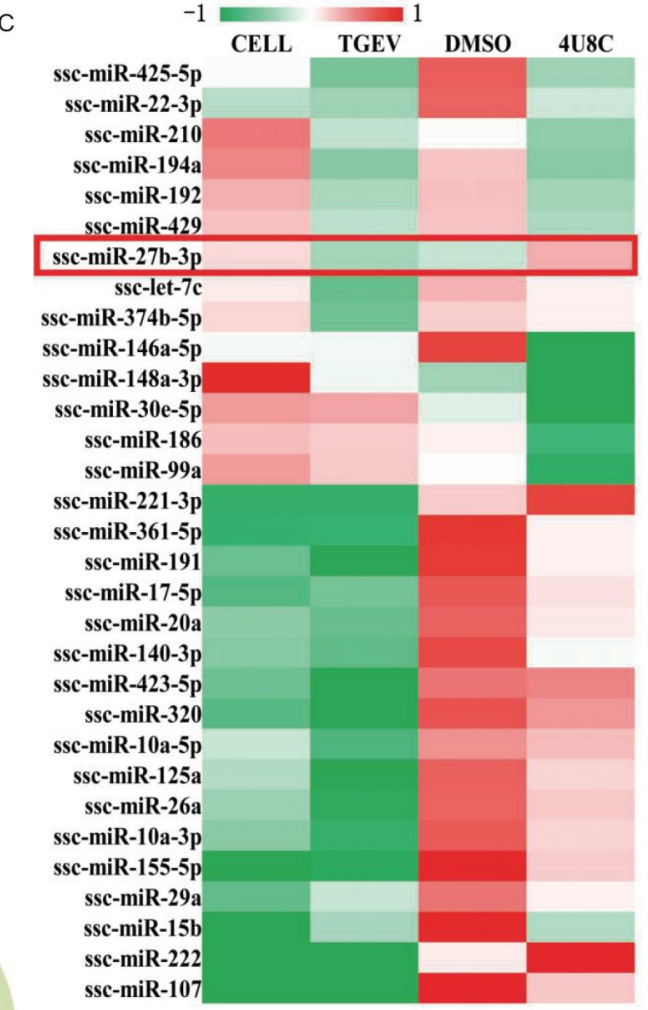

Figure 4 TGEV induced ER stress down-regulate miR-27b expression. A, The ER stress inducer Tg decreased miR-27b-3p expression in ST cells. The miR-27b-3p levels in ST cells were measured by RT-qPCR after various concentrations of Tg treatment for $24 \mathrm{~h}$. B, The miR-27b-3plevels in ST cells were measured by RT-qPCR after Tg treatment for $0,2,6,12,24,36$ and $48 \mathrm{~h}$. C, The expression of miR-27b-3p in ST cells after TGEV infection was analyzed by transcriptome sequencing. The miRNA expression levels of ST cells infected with TGEV or treated with $4 \mu 8 \mathrm{c}$ were detected by RNA-Seq. D, miRNA with common differential expression was analyzed by venn diagram.

to detect nascent pri-miR-27b with/without TGEV infection. As shown in Figure 6B, there was a significant downregulation in the transcription rate of pri-miR-27b (50\% decreased). Nuclear run-on experiments demonstrated that pri-miR-27b transcription was repressed significantly in nuclei from TGEV infected cells, suggesting that modulation of miR-27b mRNA levels is mediated by changes in transcription.

In addition, we detected the expression of pri-miR-27b and pre-miR-27b, and similar to the expression of miR-27b, the expression of pri-miR-27b and pre-mR-27 decreased with increasing TGEV concentration (Figure 6C and D). After treatment with the RNA enzyme inhibitor actinomycin D (ActD), we found that the slope of the miR-27b decrease was consistent in the TGEV-infected group and cell control group, which indicated that the degradation of miR-27b did not occur in the formation of RNA, but in the transcription level of miR-27b (Figure 6E).

\section{Xbp1 as a transcription factor inhibits the activation of miR-27b-3p promoter}

Activated IRE1 performs cytoplasmic cleavage of Xbplu transcripts, thus facilitating the synthesis of Xbp 1s (Calfon et al., 2002). Xbp1s has transcription factor activity that can activate or inhibit gene transcription (Lee et al., 2003). To verify whether Xbp1s has an impact on miR-27b transcription, we overexpressed Xbp1s plasmid. The results showed that the overexpression of Xbp1s inhibited the expression of miR-27b-3p (Figure 7A and B), while the knockdown of Xbp1s increased the expression of miR-27b-3p (Figure 7C and $\mathrm{D})$. The potential promoter region ( $2 \mathrm{~kb}$ upstream the transcriptional start site) of miR-27b was analyzed using JASPAR (Figure 7E). The JASPAR software package identified the Xbp1s as a potential regulator of miR-27b. According to the prediction, Xbp1s could bind the promoter of miR-27b at three possible sites (Figure 7F). To determine 
A
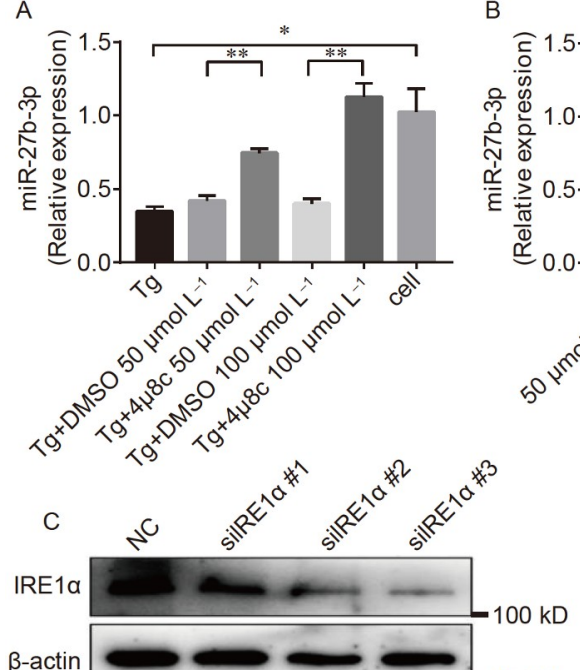

$\beta$-actin
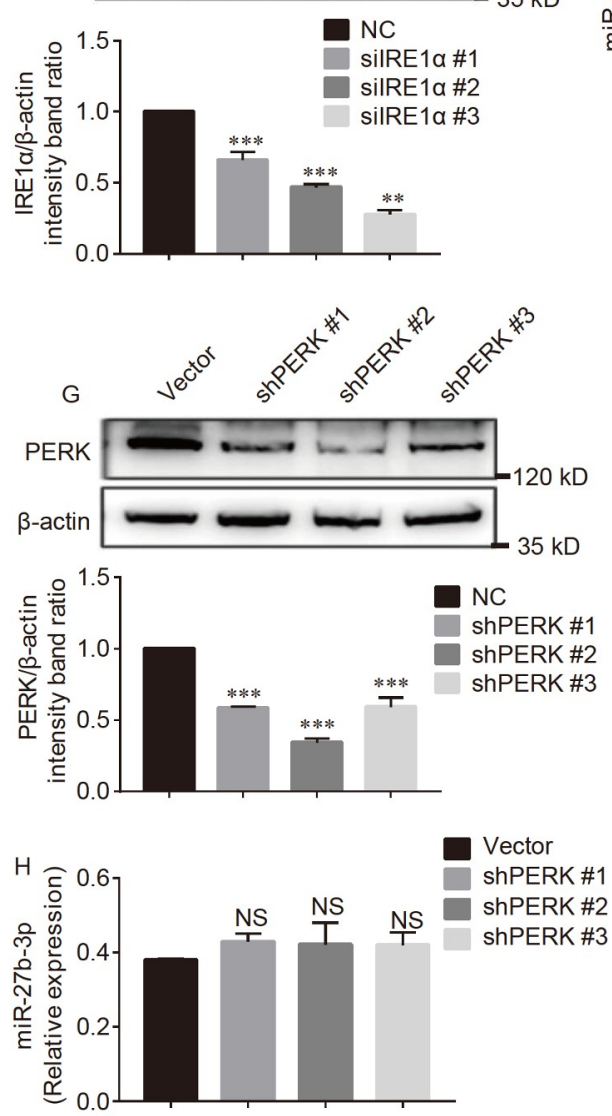

$\mathrm{D}$

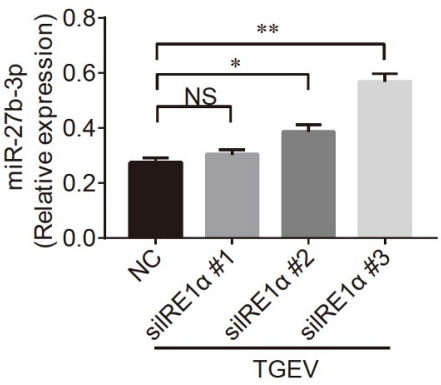

E

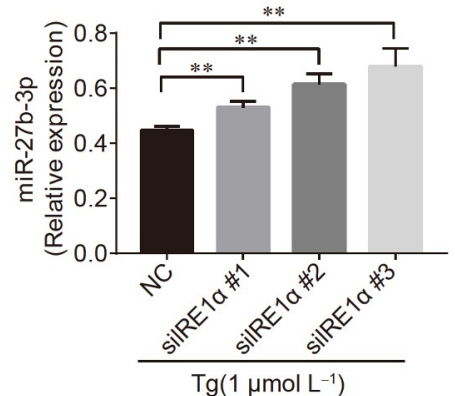

$\mathrm{F}$

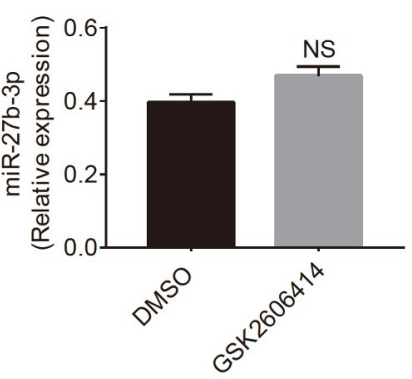

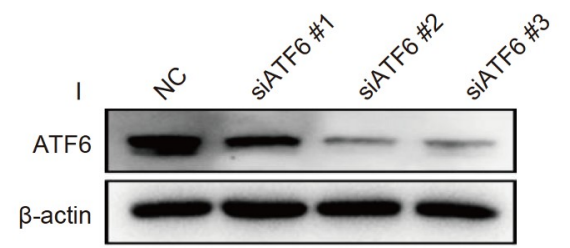
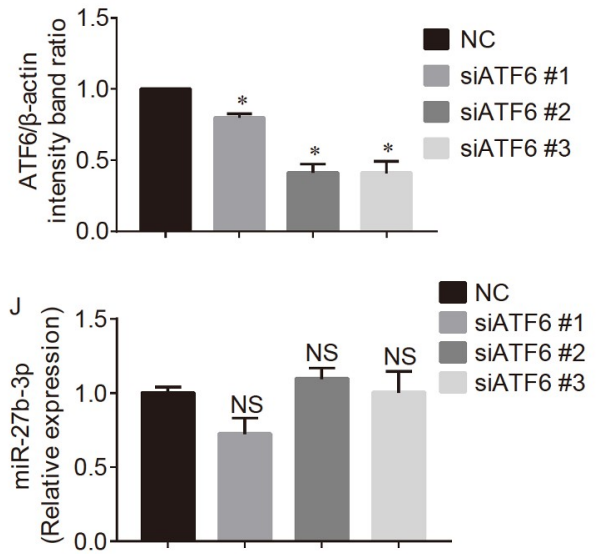

Figure 5 IRE1 $\alpha$-mediated UPR induction suppresses miR-27b-3p expression. A and B, The inhibition of IRE1 by $4 \mu 8 \mathrm{c}$ rescued the suppression of miR$27 \mathrm{~b}-3$ p by Tg (A) or TGEV (B). ST cells were pretreated with 50 or $100 \mu \mathrm{mol} \mathrm{L}^{-1} 4 \mu 8 \mathrm{c}$ for $2 \mathrm{~h}$, followed by Tg ( $1 \mu \mathrm{mol} \mathrm{L}{ }^{-1}$ ) treatment (A) or TGEV infection $(\mathrm{MOI}=1)(\mathrm{B})$. The relative expression of miR-27b-3p normalized to that of internal U6 snRNA was measured by RT-qPCR after $24 \mathrm{~h}$. C-E, Knockdown of IRE1 rescued miR-27b-3p suppression following TGEV infection or Tg treatment. ST cells were transfected with siIRE1\#1, siIRE1\#2, siIRE1\#3 or scrambled control siRNA (NC) at $100 \mathrm{nmol} \mathrm{L}^{-1}$ for $24 \mathrm{~h}$, followed by infection with TGEV for $24 \mathrm{~h}$ at a MOI of 0.1 (D) or treatment with Tg (1 $\mu$ mol L ${ }^{-1}$ ) for $24 \mathrm{~h}$ (E). Next, the cells were harvested to determine the efficiency of IRE1 knockdown (C) or miR-27b-3p expression (D and E). The results represent those from three independent experiments. F, The relative expression of miR-27b-3p in cells treated with PERK inhibitor. G, The efficiency of PERK knockdown was confirmed by Western blotting. H, Knockdown of PERK did not reverse the inhibitory effect of TGEV on miR-27b-3p. I, The efficiency of ATF6 knockdown was confirmed by Western blotting. J, Knockdown of ATF6 did not reverse the inhibitory effect of TGEV on miR-27b-3p. *, $P<0.05$; $* *, P<0.01$; $* *$, $P<0.001$; NS, not significant.

regulatory regions involved in miR-27b inhibition, we performed chromatin immunoprecipitation (ChIP) assays to confirm the validity of the binding sites. As shown in Figure $7 \mathrm{G}, \mathrm{Xbp} 1 \mathrm{~s}$ was successfully recruited by binding site 3 . 

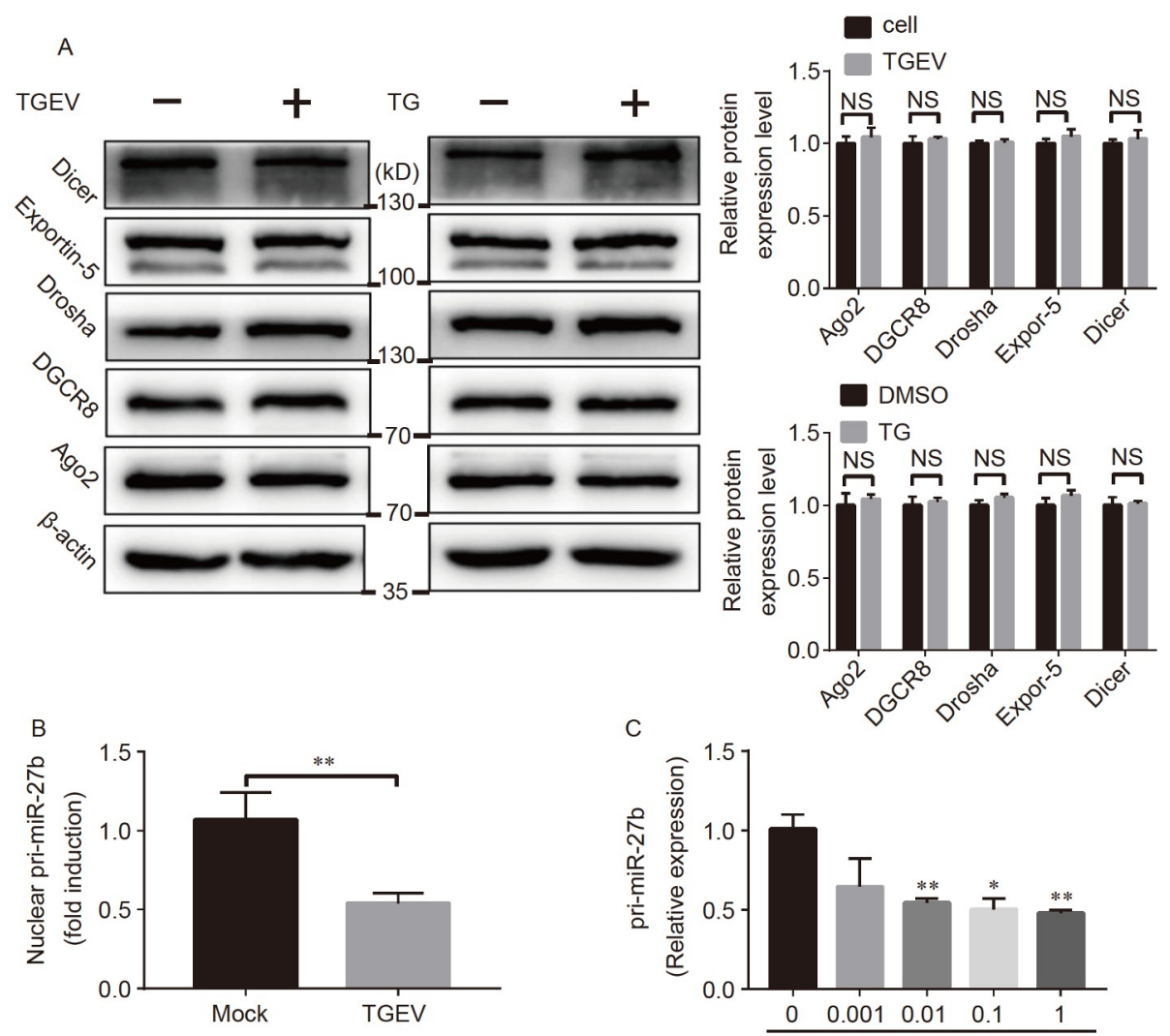

C
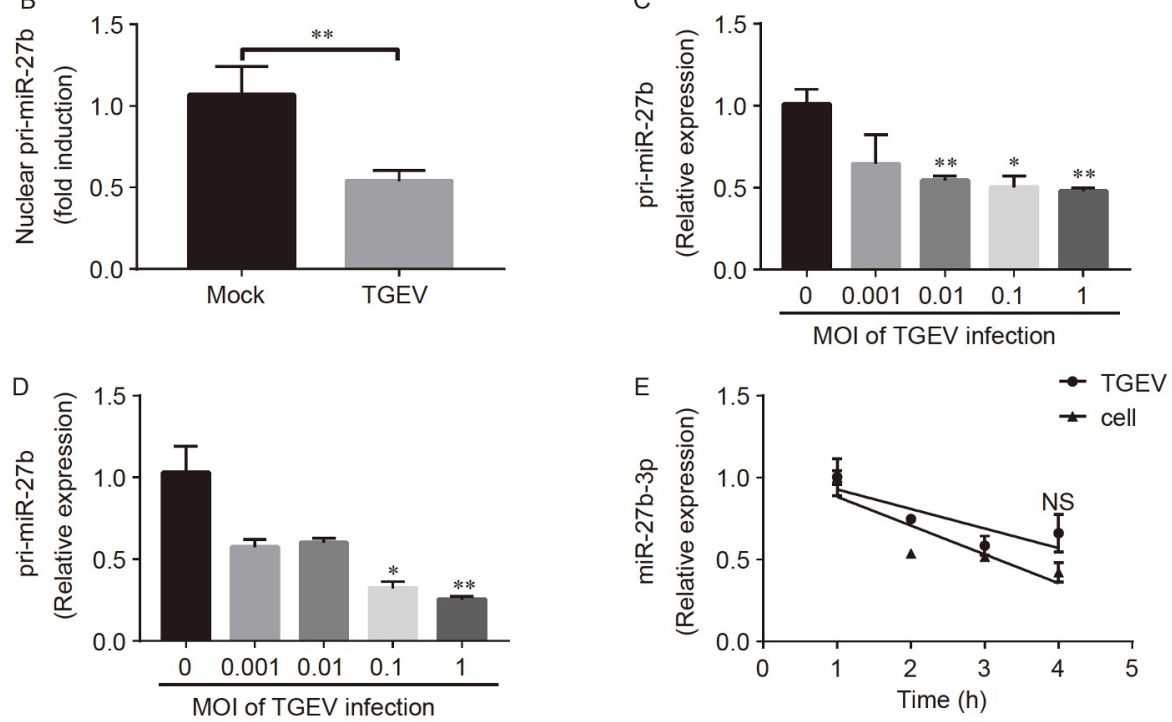

Figure 6 Mechanism of TGEV inhibiting miR-27b-3p expression. A, Expression of DGCR8, Drosha, Exportin-5, Dicer, and Ago2 in TGEV infected ST cells or under ER stress was detected by Western blotting. $\beta$-actin was used as a loading control, and the relative protein levels were quantified using the ImageJ software (right panel). Error bars represent the standard error for three independent experiments. B, Induction of the transcription rate of miR-27b transcript after TGEV infection. ST cells were infected with TGEV or mock infected, the transcription of miR-27b was assayed by using nuclear run-on analysis. Values are expressed as the mean $\pm \operatorname{SEM}(n=3)$. C and D, TGEV inhibited the expression of pri-miR-27b and pre-miR-27b. The pri-miR-27b $(\mathrm{C})$ and pre-miR-27b levels (D) in ST cells were measured by RT-qPCR at 24 hpi at different MOIs. E, miR-27b-3p expression in ST cells treated with actinomycin D (ActD) for 1, 2, 3, 4 and $5 \mathrm{~h}$.

However, Xbp1s could not bind site 1 and 2. To confirm that Xbp1s bind on the promoter of pri-miR-27b, two miR-27b promoter regions containing different Xbp1 binding sites were cloned into the pGL3 basic vector to verify the transcriptional regulation of Xbp1s to miR-27b. The transcription initiation of pri-miR-27b was successfully identified by 5'RACE (Figure 7H), and followed by sequencing (Figure S1 in Supporting Information). The two constructs were transfected into ST cells, which were then infected with TGEV or treated with Tg. Dual-luciferase reporter assays revealed that the two miR-27b promoters were down-regulated by TGEV or Tg treatment (Figure 7I). Further, the
Xbp1s overexpression vector and specific siRNA targeting Xbp1s were transfected into ST cells. The dual-luciferase analysis showed that the overexpression of Xbp1s inhibited the promoter activity of miR-27b, while the knockdown of Xbp1s activated the promoter activity of miR-27b (Figure $7 \mathrm{~J})$. These results indicate that the activated Xbp1 transcription factor inhibited the promoter of miR-27b, thus affecting the transcription of miR-27b.

\section{miR-27b may have broad-spectrum antiviral activity}

Next, we evaluated the antiviral effect of miR-27b on two 

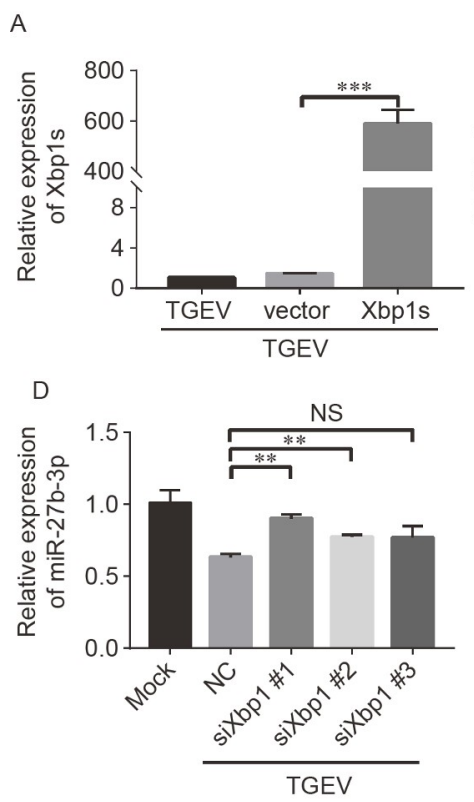

$\mathrm{F}$
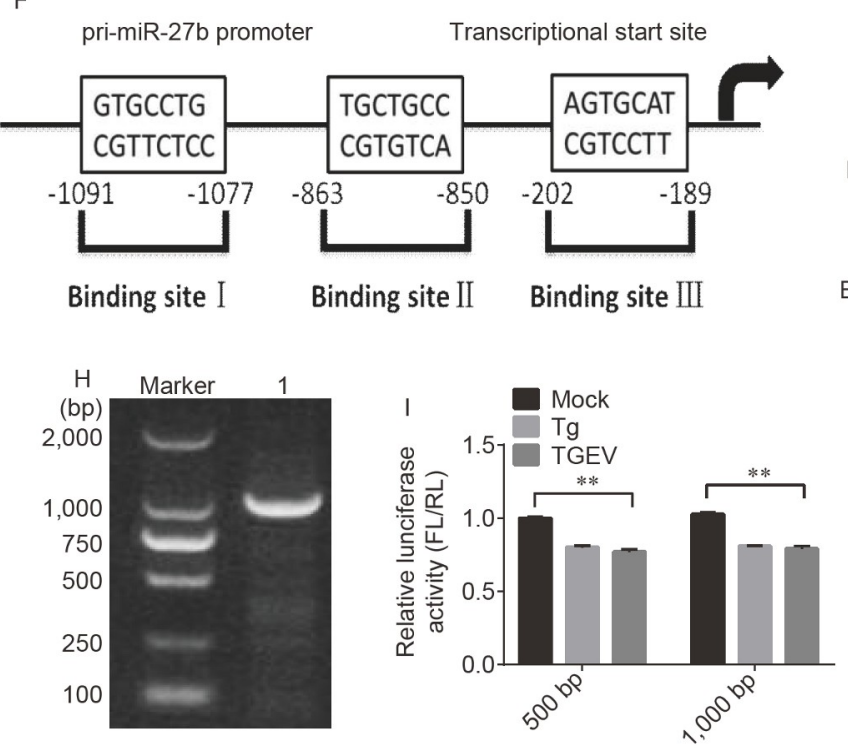

B

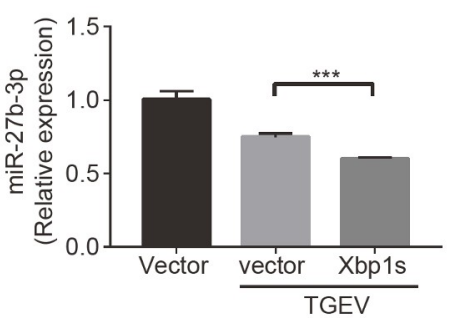

$\mathrm{E}$

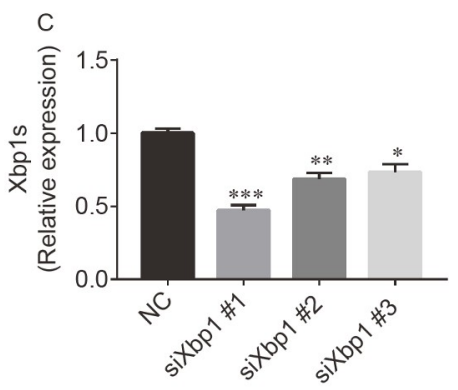

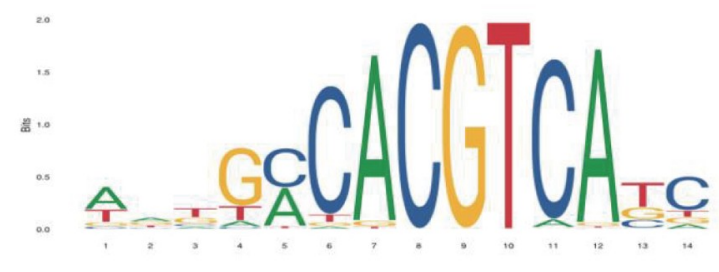
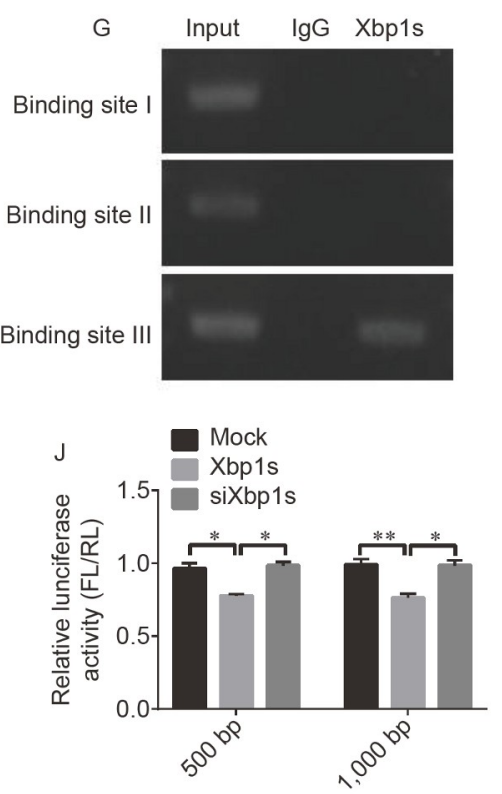

Figure 7 (Color online) Xbp1 as a transcription factor inhibited the activation of miR-27b-3p promoter. A and B, Xbp1s overexpression inhibited the expression of miR-27b-3p. Xbp1s overexpression and the expression of miR-27b-3p was verified by RT-qPCR. C and D, Xbp1s knockdown increased the expression of miR-27b-3p. Xbp1s knockdown efficiency and the expression of miR-27b-3p were verified by RT-qPCR. E-G, Determination of the Xbp1binding site on miR-27b promoter sequence. JASPAR was used for predicting Xbp1-binding site on miR-27b promoter sequence (E) and three Xbp1-binding sites were found (F). Chip assay was used to investigated whether Xbp1 directly modulated miR-27b by binding to its promoter region (G). H, Agarose gel electrophoresis showed the PCR products from 5'RACE of pri-miR-27b in ST cells. I, TGEV infection and Tg stimulation inhibited the activation of miR$27 \mathrm{~b}-3 \mathrm{p}$ promoter. J, Xbp1s overexpression inhibited the activation of miR-27b-3p promoter, while knockdown Xbp1s promoted the activation of miR-27b-3p promoter.

other common enteroviruses that cause diarrhea in pigs, PEDV (porcine epidemic diarrhea virus which belongs to the coronaviridae family) and PoRV (porcine rotavirus which belongs to the Reoviridae family). IPEC-J2 cells were transfected with the miR-27b-3p mimics or inhibitor, and cells were then inoculated with PEDV and PoRV at a MOI of 0.1. At $24 \mathrm{hpi}$, cells and supernatant were harvested for detecting viral RNA levels by RT-qPCR and viral titers by $\mathrm{TCID}_{50}$, respectively. The results showed that overexpression of miR-27b significantly inhibited PEDV and
PoRV replication as indicated by RNA levels and viral titers. While miR-27b-3p inhibitor increased PEDV and PoRV replication in IPEC-J2 cells compared with NC inhibitor (Figure 8). These results indicate that the miR-27b may have a broad-spectrum antiviral activity.

\section{DISCUSSION}

Viruses have evolved a variety of mechanisms to manipulate 
A
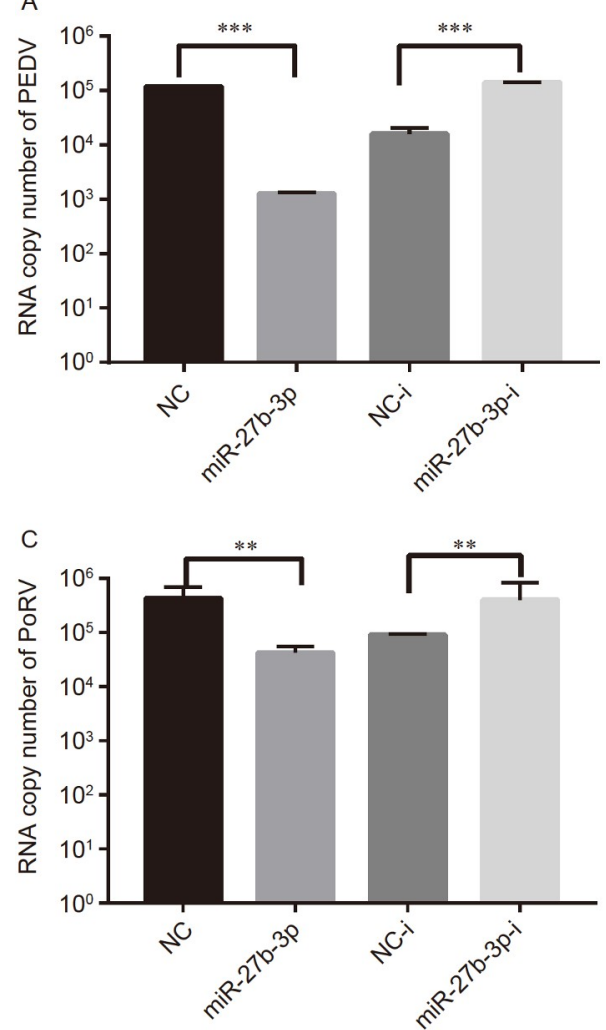

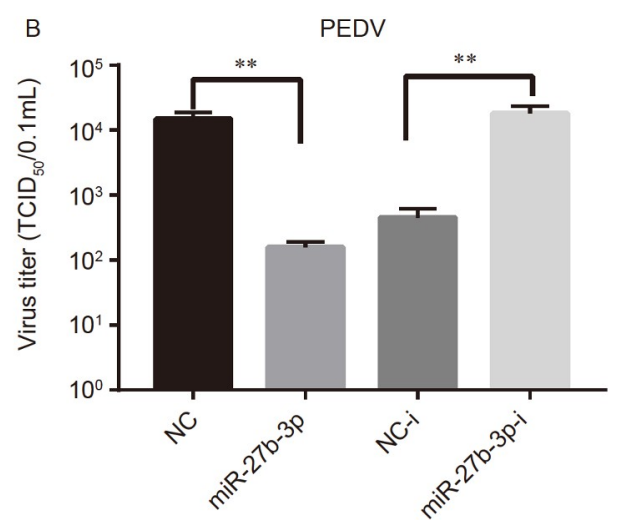

D PoRV

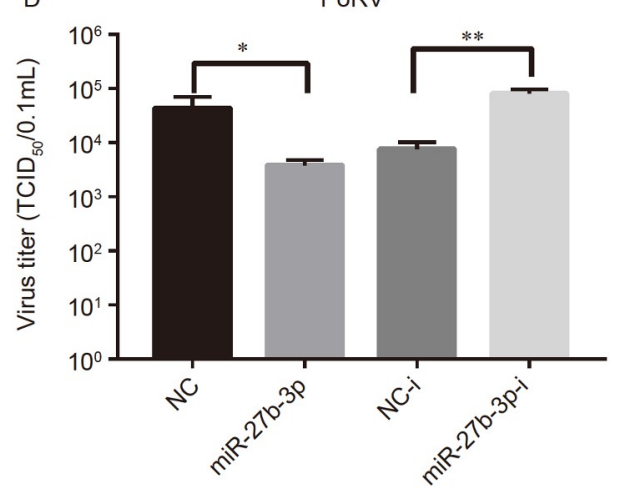

Figure 8 miR-27b may have a broad-spectrum antiviral effect. A and B, The effect of miR-27b-3p on PEDV replication. IPEC-J2 cells were transfected with miR-27b mimic or inhibitor for $24 \mathrm{~h}$, and cells were then inoculated with PEDV for $24 \mathrm{~h}$. The viral RNA levels were determined by RT-qPCR (A) and virus titers were evaluated with $\mathrm{TCID}_{50}(\mathrm{~B}) . \mathrm{C}$ and $\mathrm{D}$, The effect of miR-27b-3p on PoRV replication. IPEC-J2 cells were transfected with miR-27b mimic or inhibitor for $24 \mathrm{~h}$, and cells were then inoculated with PoRV for $24 \mathrm{~h}$. The viral RNA levels were determined by RT-qPCR (C) and virus titers were evaluated with $\mathrm{TCID}_{50}(\mathrm{D})$.

host cells and evade antiviral defenses. The most extensively studied miRNA-virus interactions demonstrate that RNA viruses can use cellular miRNAs to regulate their replication (Forster et al., 2015; O'Neill et al., 2011). In this study, we found that miR-27b can inhibit the replication of TGEV by directly targeting porcine SOCS6. As a coronavirus, TGEV has evolved a mechanism to antagonize the antiviral effect of miR-27b. Mechanistically, TGEV infection activates ER stress and inhibits the activation of the miR-27b promoter through the transcription factor activity of Xbp1s in the IRE1 pathway, thereby inhibiting its expression and antagonizing the antiviral effect of miR-27b (Figure 9).

In mammals, miRNAs can play different roles in viral infection through their capacity to regulate both host and viral genes (Lecellier et al., 2005; Mahajan et al., 2009). Recent studies have demonstrated that the expression level of specific miRNAs change during infection and affect the production and infectivity of viruses (Mahajan et al., 2009). miR-32 was shown to target open reading frame 2 of primate foamy virus type-1 (PFV-1), thereby inhibiting the translation of virus mRNA and limiting the accumulation of retroviruses in cultured human cells (Lecellier et al., 2005). Another report demonstrated that several cellular miRNAs, including miR-28, miR-125b, miR-150, miR-223 and miR-
382, can inhibit HIV-1 replication by targeting sequences in the 3 '-end of HIV-1 RNA (Huang et al., 2007). Both miR-27a and miR-27b have been reported to exert an antiviral effect against murine cytomegalovirus (Buck et al., 2010). In the present study, we showed that overexpression of miR-27b inhibited the replication of coronavirus (PEDV and TGEV) and PoRV, suggesting that miR-27b has a broad-spectrum antiviral activity (Figures 1 and 8). Overall, these data indicate that certain cellular miRNAs are form part of the host's innate antiviral defense.

In the context of infection, viruses can alter the expression of host miRNAs, either in cell culture or in the host (Mahajan et al., 2009). For example, miR-155 was up-regulated within $2 \mathrm{~h}$ after poly (I:C) treatment (O'Connell et al., 2007) or upon infection with Epstein-Barr virus (Cameron et al., 2008a; Cameron et al., 2008b; Gatto et al., 2008). A previous study revealed that miR-221-5p was up-regulated by PEDV infection (Zheng et al., 2018). Another study revealed that vesicular stomatitis virus infection induced miR-221 in an ELF4-dependent manner (Du et al., 2018). Here, infection of ST cells or piglets with TGEV induces the down-regulation of an antiviral cellular miRNA, miR-27b. To date, the downregulation of miR-27 during viral infection has only been reported in mouse cytomegalovirus (MCMV) (Buck et al., 


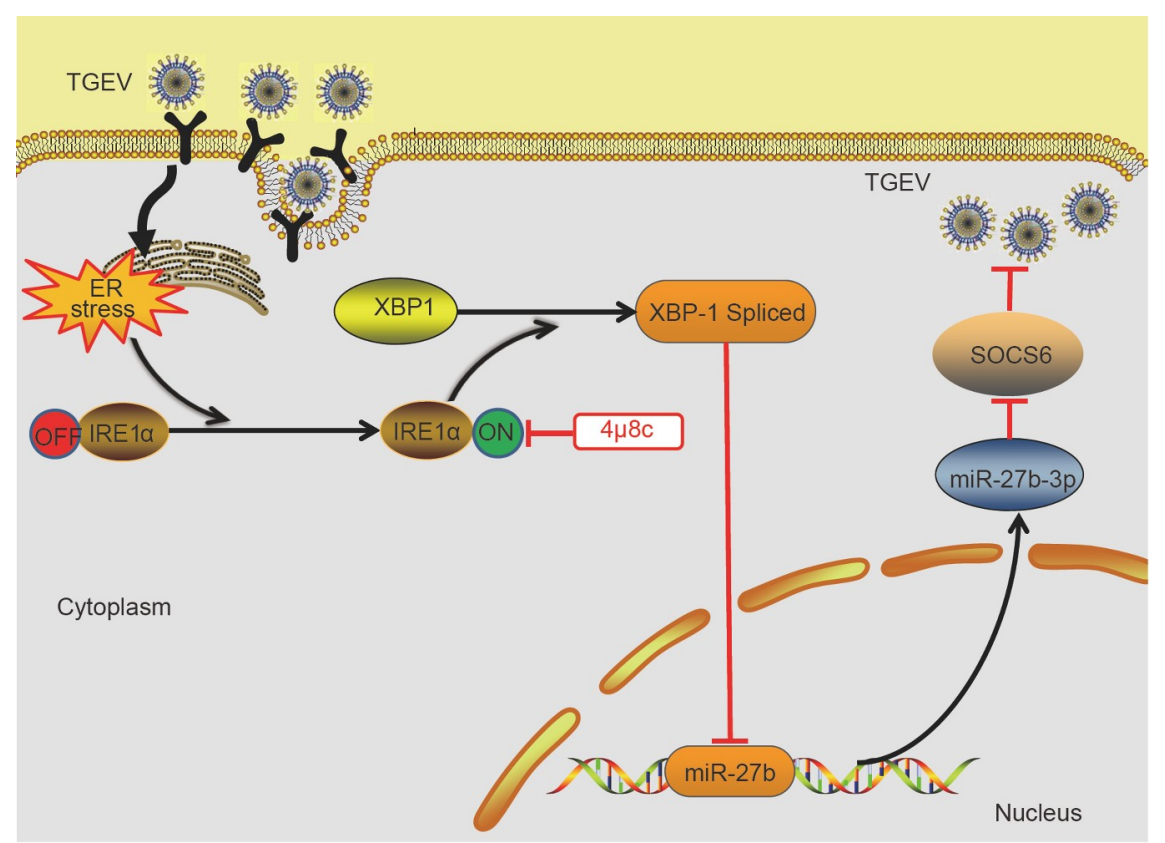

Figure 9 (Color online) The mechanism of TGEV antagonize the antiviral effect of miR-27b. miR-27b overexpression can inhibit TGEV replication by directly targeting porcine SOCS6. As a coronavirus, TGEV has evolved a mechanism to antagonize the antiviral effect of miR-27b. The mechanism is TGEV infection induced ER stress and activated IRE1 pathway. The activation of IRE1 leads to the activation of downstream Xbp1. Xbp1s as a transcription factor inhibits the activation of miR-27b-3p promoter, resulting in the inhibition of miR-27b-3p expression, thus antagonizing the antiviral effect of miR-27b.

2010). Therefore, further research is needed to determine whether the down-regulation of miR-27b is a part of the host response or a viral strategy involving viral factors.

IRE1 $\alpha$ as the most highly conserved UPR sensor, it has kinase and RNase activity and plays a key role in restoring ER homeostasis (Credle et al., 2005; Hassler et al., 2012). Many viruses have evolved strategies involving IRE1 to inhibit signaling pathways and thus promote infection. The inhibition of IRE1 $\alpha$ signaling or siRNA knockdown reduces the replication rate of IAV, JEV and hepatitis $\mathrm{C}$ virus (HCV) (Bhattacharyya et al., 2014; Fink et al., 2017; Hassan et al., 2012). IRE1 $\alpha$ promotes JEV virus replication by regulating the IRE1-dependent decay (RIDD) pathway and regulating virus RNA translation (Bhattacharyya et al., 2014). In this study, we identified another mechanism by which TGEV enhances viral infection through IRE1: reducing the abundance of endogenous miR-27b-3p. In previous studies, we observed that IRE1 mediates the decrease in miR-30a-5p levels in Tg-treated ST cells (Ma et al., 2018). Therefore, the down-regulation of miR-27b-3p by IRE1 inhibitor is not specific. This finding is consistent with previous studies showing that IRE1 that is activated after TGEV infection can regulate the expression of miRNA subsets, including miR125b, miR-150, miR-17 and miR-30a-5p, in response to ER stress (Fink et al., 2017; Hassler et al., 2012; Heindryckx et al., 2016). However, the specificity and mechanism of IRE1 mediated miRNAs regulation are still unclear, which is worthy of further study.

Although there is growing evidence showing the co-reg- ulation of mature miRNAs and signal transducers in the UPR signaling pathway (Berry et al., 2018; Zhang et al., 2020), little is known about the processing of pri-miRNAs and premiRNAs during ER stress. Our study showed that the expression of most miRNAs in ST cells changed under ER stress (Figure 4C), suggesting that ER stress is involved in regulating the expression of miRNAs. We tried to determine whether the change in miRNA expression can be attributed to ER stress in miRNA processing. Surprisingly, the expression of several key components of the biogenetic mechanism of miRNA, including the pri-miRNA processing factors DGCR8 and Drosha, pre-miRNA transfer factor Exportin-5, pre-miRNA processing factors Dicer and Ago2, did not fluctuate during ER stress (Figure 6A). In addition, there was no significant change in the mRNA or protein levels of these genes $24 \mathrm{~h}$ after $\mathrm{Tg}$ treatment, indicating that the change in miRNA expression during ER stress was not caused by pri-miRNA or pre-miRNA processing. It may be regulated by unknown factors that affect the stability of mature miRNA during ER stress. In recent years, an increasing number of studies have reported that the stability of miRNAs is regulated by transcription factors or related to their own sequences. Yu and Hecht found that transposable proteins and DNA/RNA-binding proteins bind to miR-122a and increase their stability in vivo (Yu and Hecht, 2008). Bail et al. (2010) reported that miR-382, which contributes to the incubation period of the HIV-1 provirus, is unstable in cells, and the $3^{\prime}$ end of this miRNA is necessary for its instability.

In this study, we demonstrated that miR-27b-3p 
suppressed TGEV replication, while TGEV infection downregulated miR-27b-3p expression in vitro and in piglets. We further found that when TGEV mediated ER stress occurs, IRE1 acts as RNase, unconventionally splicing the mRNA encoding a potent transcription factor $\mathrm{Xbp} 1$, and $\mathrm{Xbp} 1$ acts as a transcription factor, inhibiting the transcription of miR$27 b$ (Figure 7), resulting in the inhibition of miR-27b-3p production, thus antagonizing the antiviral effect of miR$27 \mathrm{~b}$. The results of this study revealed a new mechanism of coronavirus escaping from the host antiviral immune response.

\section{MATERIALS AND METHODS}

\section{Cell culture and virus}

ST cells were maintained under standard culture conditions with Dulbecco's Minimal Essential Medium (DMEM; Gibco, USA) plus 10\% fetal bovine serum (FBS; Gibco), $100 \mathrm{IU}$ of penicillin, and $100 \mathrm{mg} \mathrm{mL}^{-1}$ streptomycin at $37^{\circ} \mathrm{C}$ and $5 \%$ $\mathrm{CO}_{2}$. The $\mathrm{ST}$ cell-adapted TGEV strain $\mathrm{H} 87$ derived from the virulent strain H16 (GenBank accession No. FJ755618) was propagated as previously described (Xue et al., 2018). For TGEV infection, ST cells were mock infected with DMEM or infected with TGEV $\mathrm{H} 87$ at the desired MOI. After a $2 \mathrm{~h}$ incubation at $37^{\circ} \mathrm{C}$, cells were washed and cultured in DMEM supplemented with 1\% DMSO and $0.3 \%$ trypsin $(0.25 \%$; Gibco) until harvested.

The porcine small intestinal epithelial cell line IPEC-J2 was grown in DMEM-nutrient mixture F-12 (Ham) (1:1) (DMEM-F12) (Gibco), supplemented with 5\% FBS, $5 \mathrm{ng} \mathrm{mL}^{-1}$ epidermal growth factor (Gibco), $5 \mathrm{~g} \mathrm{~mL}^{-1}$ insulin-transferring selenium supplements (Gibco) and 1\% penicillin-streptomycin.

\section{Cloning and construction of plasmids}

The promoter region of pig miR-27b was amplified by genomic PCR (upper primer 1,000 bp, 5'CGGGGTACCTCTCACTGTGATCTTTCCTGC-3'; upper primer $500 \mathrm{bp}$, 5'-CGGGGTACCAGCCTCTGGGCACTTACAAGC-3'; lower primer, 5'-CCCAAGCTTACAGTTCTGGAGGATGAGAGC-3') and the PCR fragment was inserted into the KpnI-HindIII site of PGL3-Basic vector. The recombinant expression plasmids p27b (-561/-61) luc and p27b (-1,061/-61) luc were sequenced, and no unexpected mutations occurred. The TargetScan web server (version 7.1; http://www.targetscan.org) was used to predict SOCS6 3'UTRs as potential targets of miR-27b-3p. The 3'UTR of porcine SOCS6 (GenBank accession number GQ421918.1) was amplified and inserted into the pmirGLO luciferase reporter vector (Promega, USA) using the NheI and XbaI restriction sites. The mutant types of SOCS6
3'UTR vectors were constructed by mutating seven seed nucleotides using a site-directed mutagenesis kit (Stratagene, USA) according to the manufacturer's instructions. The primers are listed in Table 1.

\section{5' rapid amplification of CDNA ends (RACE)}

$5^{\prime}$ RACE was performed to identify the transcription initiation sites in pri-miR-27b with a SMARTer ${ }^{\mathrm{TM}} \mathrm{RACE} 5^{\prime} / 3^{\prime}$ kit (Clontech, USA). Briefly, total RNA was isolated from ST cells. RACE-ready cDNA was synthesized according to the manufacturer's instructions. The obtained cDNA was purified on a $1.2 \%$ agarose gel and ligated to the linearized pRACE vector, and sequenced.

\section{RNA preparation and small RNA sequencing}

Confluent ST cells in $100 \mathrm{~mm}$ cell culture dish were treated with $4 \mu 8 \mathrm{C}$ for $2 \mathrm{~h}$ or left untreated, then cells were infected with TGEV for $24 \mathrm{~h}$ at a MOI of 1.0. Meanwhile, the mock infection was carried out. Each group had three duplicates. At 24 hpi, total RNA was extracted with Trizol reagent (Invitrogen, USA). Total RNA of each sample was qualified by Agilent 2100 Bioanalyzer (Agilent Technologies, USA), NanoDrop (Thermo Fisher Scientific, USA) and 1\% agrose gel. $2 \mu \mathrm{g}$ total RNA with RIN value above 7.5 was used for the following library preparation. Next-generation sequencing library preparations were constructed according to the manufacturer's protocol (NEBNext ${ }^{\circledR}$ Multiplex Small RNA library Prep Set for Illumina $\left.{ }^{\circledR}\right)$. Briefly, $3^{\prime}$ SR Adaptor for Illumina was ligated to the small RNA using $3^{\prime}$ Ligation Enzyme. To prevent adaptor-dimer, the excess of 3'SR Adaptor were hybrid with SR RT Primer for Illumina. 5'SR Adaptor for Illumina was ligated to the small RNA using 5'Ligation Enzyme and the first-strand cDNA was synthesized using ProtoScript II Reverse Transcriptase. Each sample was then amplified by PCR for 12 cycles using P5 and $\mathrm{P} 7$ primers, with both primers carrying sequences which can anneal with flow cell to perform bridge PCR and P7 primer carrying a six-base index allowing for multiplexing. The PCR products of $\sim 140$ bp were recovered and cleaned up using PAGE, validated using an Agilent 2100 Bioanalyzer (Agilent Technologies), and quantified by Qubit 2.0 Fluorometer (Invitrogen).

\section{miRNA and siRNA transfections}

RNA oligos including miRNA mimic, miRNA inhibitor, siRNA and the corresponding negative control were synthesized by Genepharma (Suzhou, China). The day before transfection, cells were trypsinized and seeded in medium without antibiotics in 24-well plates. ST cells were transfected with miRNA mimics or inhibitors (GenePharma) 
Table 1 Sequences of primers used in the present study

\begin{tabular}{|c|c|}
\hline Primer & Sequences $\left(5^{\prime}-3^{\prime}\right)$ \\
\hline miR-27b-3p-qPCR-F & CGCTTCACAGTGGCTAAGTTCTGC \\
\hline Uni-miR-qPCR-R & GCGAGCACAGAATTAATACGACTCAC \\
\hline GAPDH-qPCR-F & ATGGGGAAGGTGAAGGTCGG \\
\hline GAPDH-qPCR-R & TCCTGGAAGATGGTGATGGG \\
\hline pre-miR-27b-qPCR -F & AGTGACTGGTTTCCGCTTTG \\
\hline pre-miR-27b-qPCR -R & GAGTCGGCAATTCAGTTGAGC \\
\hline pri-miR-27b-qPCR -F & GTGACTGGTTTCCGCTTTGTTC \\
\hline pri-miR-27b-qPCR -R & ACAGAGTGCATCGTCCTTG \\
\hline U6-qPCR-F & CTCGCTTCGGCAGCACA \\
\hline U6-qPCR-R & AACGCTTCACGAATTTGCGT \\
\hline SOCS6-qPCR-F & TCAAGAGTTCCGAGTCTCAGG \\
\hline SOCS6-qPCR-R & ССТССТССAAAGGCACAGTGT \\
\hline TGEV-qPCR-F & GCTTGATGAATTGAGTGCTGATG \\
\hline TGEV-qPCR-R & CCTAACCTCGGCTTGTCTGG \\
\hline Xbp1-F & AAACAGAGTAGCAGCTCAGACTGC \\
\hline Xbp1-R & GAATCTCTAAGACTAGGGGCTTTGTA \\
\hline SOCS6-3' UTR-Nhe I-F & CTAGCTAGC AAGATGATGGGAACCCTGCAT \\
\hline SOCS6-3' UTR-Xba I-R & TGCTCTAGA ATGTAAGGCCATTTATTAAAA \\
\hline ChIP-BS1-F & TTCAGACAGGTGTCTAACTGG \\
\hline ChIP-BS1-R & CACAGTTCTGGAGGATGAGAG \\
\hline ChIP-BS2-F & TACTAGACAGGCAGCCCGTTG \\
\hline ChIP-BS2-R & GAGTCGTAGAGCTGTGTTGAC \\
\hline ChIP-BS3-F & TCTCACTGTGATCTTTCCTGC \\
\hline ChIP-BS3-R & GTCTCCTCGTGTTCTCGCTC \\
\hline
\end{tabular}

using Lipofectamine RNAiMAX (Life Technologies, USA). Cells were incubated $24 \mathrm{~h}$ at $37^{\circ} \mathrm{C}$ and $5 \% \mathrm{CO}_{2}$ prior to infection with TGEV, followed by real-time RT-PCR analysis and Western blot analysis as described below. Supernatant from the infected cells was collected at $24 \mathrm{hpi}$, and the viral titer was measured by $\mathrm{TCID}_{50}$. For siRNA transfection, ST cells were grown to $70 \%-80 \%$ confluence before transfection with synthetic oligonucleotides using the Lipofectamine 2000 reagent (Invitrogen) according to the manufacturer's protocol. After $6 \mathrm{~h}$, transfection mix was replaced with complete medium. The sequence of siRNAs was listed in Table 2.

\section{Virus titration}

ST cells grown in 24-well culture plates (CoStar, USA) were treated with chemicals $(\mathrm{Tg}, 4 \mu 8 \mathrm{C}$, GSK2606414 or DMSO control) and infected with TGEV for $24 \mathrm{~h}$, or transfected with siATF6, siIRE1, shPERK for $24 \mathrm{~h}$, and then infected with TGEV at a MOI of 0.1 . At $24 \mathrm{hpi}$, the culture supernatants were collected, and the cells were subjected to three freeze-thaw cycles. The cultures were serially diluted (10fold) from $10^{-1}$ to $10^{-10}$ and added to confluent ST cells in 96-well plates (CoStar). After $72 \mathrm{~h}$ of incubation, the supernatant was removed, cells were fixed with $4 \%$ polyformaldehyde and the viral antigen was detected using immunofluorescence. Viral titers were calculated using the Reed-Muench method and expressed as $\mathrm{TCID}_{50} / 0.1 \mathrm{~mL}$.

\section{miRNA and pri-miRNA quantification by quantitative real-time PCR}

Total RNA was extracted from cells using RNA Rapid extraction kit (Bioer, Hangzhou, China) and reverse transcribed using the PrimeScript ${ }^{\mathrm{TM}}$ II 1st Strand cDNA Synthesis Kit (TaKaRa, Dalian, China). For miRNA analyses, RNA was reverse transcribed by using an miRNA first-strand cDNA synthesis kit (Sangon Biotech, Shanghai, China). The primers for real-time quantitative PCR assay were shown in Table 1. The cDNA was used as a template in the SYBR Green PCR assay (Roche, Germany). The abundance of individual mRNA transcripts in each sample was assayed thrice using GAPDH and U6 as an internal control. The expression pattern of each gene was analyzed by RT-qPCR using a Roche LightCycler 480 Real Time PCR System as previously described (Xue et al., 2018). PCRs were subjected 
Table 2 Sequences of miRNA mimics, inhibitors, and siRNAs

\begin{tabular}{cc}
\hline Small RNA & Sequence $\left(5^{\prime}-3^{\prime}\right)$ \\
\hline miR-27b-3p & UUCACAUGGCUAAGUUCUGC \\
miR-27b-3p inhibitor & GCAGAACUUAGCCACUGUGAA \\
siIRE1\#1 & GCACAGACCUGAAGUUCAATT \\
siIRE1\#2 & GGAGGUUAUCGACCUGGUUTT \\
siIRE1\#3 & CCAUCAUCCUGAGCACCUUTT \\
NC-sense & UUCUCCGAACGUGUCACGUTT \\
NC-antisense & ACGUGACACGUUCGGAGAATT \\
\hline
\end{tabular}

to $15 \mathrm{~min}$ of $95^{\circ} \mathrm{C}$ hot-start enzyme activation, and 40 cycles of $95^{\circ} \mathrm{C}$ denaturation for $15 \mathrm{~s}, 60^{\circ} \mathrm{C}$ annealing for $30 \mathrm{~s}$, and $72^{\circ} \mathrm{C}$ elongation for $30 \mathrm{~s}$, including subsequent melting curve analysis. The data was analyzed using the $\Delta \Delta C_{\mathrm{t}}$ method (Bookout et al., 2006) using U6 snRNA as an endogenous reference, and the mock-infected sample as a calibrator.

\section{Nuclear run-on assays}

Nuclear run-on assays were performed to examine nacent pri-miR-27b with/without TGEV infection. ST cells were seeded onto 24 -well plates at a cell density of $5.0 \times 10^{5}$ cells/ well and infected with TGEV for $24 \mathrm{~h}$ or mock infected. Non-radioactive nuclear run-on assays were performed as described previously (Rivera-Ramírez et al., 2019).

\section{ChIP assay}

The ChIP assay was performed using a commercial kit (Beyotime, Shanghai, China) according to the manufacturer's instructions. An antibody against Xbp1s was used to immunoprecipitate Xbp1s-chromatin complexes. AntiIgG (Santa Cruz Biotechnology, USA) served as a negative control. The ChIP products were amplified by PCR and then separated on $1.5 \%$ agarose gels. The primers for amplification are listed in Table 1.

\section{Northern blot analysis}

Northern blots were performed using a NorthernMax ${ }^{\mathrm{TM}}$-Gly kit (Invitrogen) according to the manufacturer's instructions. Briefly, total RNA was isolated from cells using the RNeasy mini kit (QIAGEN, Germany). The RNA samples $(15 \mu \mathrm{g}$ each) were resolved on $0.9 \%$ denaturing formaldehyde agarose gel, transferred to BrightStar ${ }^{\mathrm{TM}}$-Plus Positively Charged Nylon Membrane, and probed with ${ }^{32} \mathrm{P}$-labeled antisense riboprobes specific to miR-27b at $68^{\circ} \mathrm{C}$ (Table 1). U6 was taken as loading control. The bands were scanned with a STORM 860 Molecular Imager and Image Quant software (Molecular Dynamics).

\section{Dual-luciferase reporter assays.}

The 1,000 bp upstream of miR-27b promoter was amplified by PCR and cloned into pGL3-Basic using primers: forward 5'-GATCTCGAGGTTCCTTACTGGCTTGCT-3' and reverse 5'-CTGAAGCTTTTGTCAGACTGAAGAATC-3'. Furthermore, the miR-27b reporter plasmid and pRL-TK were cotransfected with either siXbp-1 or Xbp-1 expression vectors for $24 \mathrm{~h}$ and treated with Tg or TGEV for the time as indicated. Luciferase assays were performed using the DualLuciferase Reporter Assay System (Promega) according to the manufacturer's protocol. Lysates were then centrifuged to remove cellular debris and $100 \mu \mathrm{L}$ of the supernatants were loaded into an automated luminometer (PerkinElmer EnVision, UK). Then the instrument performed a sequential auto-injection of $100 \mu \mathrm{L}$ of Luciferase Assay Reagent II (substrate for firefly luciferase) and $100 \mu \mathrm{L}$ of Stop and Glow Reagent (stop solution for firefly luciferase containing the substrate for Renilla luciferase). The mean of the luciferase activities measured for $10 \mathrm{~s}$ each was used to calculate ratios between firefly and Renilla luciferases.

\section{Western blot analysis}

After rinsed with ice-cold PBS, ST cells were lysed with Nonidet P-40 (NP-40) lysis buffer (Beyotime) supplemented with $1 \mathrm{mmol} \mathrm{L}^{-1}$ phenylmethyl sulphonylfluoride (PMSF). Protein concentrations were assayed using BCA Protein Assay Reagent (Pierce, USA). Equivalent amounts of proteins were subjected to $8 \%-12 \%$ SDS-PAGE and subsequently transferred onto a polyvinylidene difluoride (PVDF) membranes (Millipore, USA). The membranes were blocked in PBS buffer with 5\% non-fat dry milk at room temperature for $1 \mathrm{~h}$, and then subsequently incubated with primary antibodies overnight at $4^{\circ} \mathrm{C}$, followed by HRP-conjugated secondary antibodies incubation at room temperature for $1 \mathrm{~h}$. The signal was detected by increased chemiluminescence (ECL) reagents (Pierce). The intensity of each band was analyzed using ImageJ software. The primary antibodies used were as follows: $\beta$-actin (1:5,000; Sigma, USA), IRE1 (1:500; Santa Cruz Biotechnology), the monoclonal antibody against TGEV $\mathrm{N}$ protein was prepared in our laboratory.

\section{Immunofluorescence assay}

IFA was performed as previously described. ST cells were fixed with $4 \%$ paraformaldehyde for $30 \mathrm{~min}$ at room temperature followed by fixed cells were permeabilized with $0.2 \%$ Triton $\mathrm{X}-100$ and then blocked with blocking buffer for $2 \mathrm{~h}$ at $37^{\circ} \mathrm{C}$. Cells were incubated with an anti-TGEV N protein monoclonal antibody $(1: 1,000)$ stocked in the laboratory or an anti hemagglutinin (anti-HA) monoclonal antibody $\left(1: 5,000\right.$; Sigma) at $37^{\circ} \mathrm{C}$ for $2 \mathrm{~h}$. The cells were 
then labeled with an Alexa Fluor 546 goat anti-mouse immunoglobulin $\mathrm{G}$ (IgG) antibody (1:500; Thermo Fisher Scientific) for $1 \mathrm{~h}$ at $37^{\circ} \mathrm{C}$. DAPI (Sigma) was used for the staining of cellular nuclei. The stained cells were visualized using an AMG EVOS F1 fluorescence microscope (Thermo Fisher Scientific)

\section{Statistical analysis}

All data are expressed as means \pm standard errors of the means (SEMs) from three independent experiments and were analyzed through a one-way analysis of variance in GraphPad Prism 7. Differences were considered significant if the $P$ value was $<0.05$. $P$ values are indicated as follows: *, $P<0.05$; **, $P<0.01 ; * * *, P<0.001$.

\section{Compliance and ethics The author(s) declare that they have no conflict} of interest.

\begin{abstract}
Acknowledgements This work was supported by the Heilongjiang Postdoctoral fund (LBH-Z18207), the National Natural Science Foundation of China (31802198), the Fundamental Research Funds for the Provincial Universities (2018-KYYWF-0553), the National Key Research and Development Program of China (2017YFC0908001) and the Spark Research Fund from the Fourth Affiliated Hospital of Harbin Medical University (HYDSYXH201914).
\end{abstract}

\section{References}

Bail, S., Swerdel, M., Liu, H., Jiao, X., Goff, L.A., Hart, R.P., and Kiledjian, M. (2010). Differential regulation of microRNA stability. RNA 16, 1032-1039.

Bartel, D.P. (2004). MicroRNAs: genomics, biogenesis, mechanism, and function. Cell 116, 281-297.

Berry, C., Lal, M., and Binukumar, B.K. (2018). Crosstalk between the unfolded protein response, microRNAs, and insulin signaling pathways: in search of biomarkers for the diagnosis and treatment of type 2 diabetes. Front Endocrinol 9, 210.

Bhattacharyya, S., Sen, U., and Vrati, S. (2014). Regulated IRE1-dependent decay pathway is activated during Japanese encephalitis virus-induced unfolded protein response and benefits viral replication. J Gen Virol 95, 71-79.

Bookout, A.L., Cummins, C.L., Mangelsdorf, D.J., Pesola, J.M., and Kramer, M.F. (2006). High-throughput real-time quantitative reverse transcription PCR. Curr Protoc Mol Biol 73.

Buck, A.H., Perot, J., Chisholm, M.A., Kumar, D.S., Tuddenham, L., Cognat, V., Marcinowski, L., Dolken, L., and Pfeffer, S. (2010). Posttranscriptional regulation of miR-27 in murine cytomegalovirus infection. RNA 16, 307-315.

Calfon, M., Zeng, H., Urano, F., Till, J.H., Hubbard, S.R., Harding, H.P., Clark, S.G., and Ron, D. (2002). IRE1 couples endoplasmic reticulum load to secretory capacity by processing the XBP-1 mRNA. Nature 415 , 92-96.

Cameron, J.E., Fewell, C., Yin, Q., McBride, J., Wang, X., Lin, Z., and Flemington, E.K. (2008a). Epstein-Barr virus growth/latency III program alters cellular microRNA expression. Virology 382, 257-266.

Cameron, J.E., Yin, Q., Fewell, C., Lacey, M., McBride, J., Wang, X., Lin, Z., Schaefer, B.C., and Flemington, E.K. (2008b). Epstein-Barr virus latent membrane protein 1 induces cellular microRNA miR-146a, a modulator of lymphocyte signaling pathways. J Virol 82, 1946-1958.

Credle, J.J., Finer-Moore, J.S., Papa, F.R., Stroud, R.M., and Walter, P. (2005). On the mechanism of sensing unfolded protein in the endoplasmic reticulum. Proc Natl Acad Sci USA 102, 18773-18784.

Ding, L., Ni, J., Yang, F., Huang, L., Deng, H., Wu, Y., Ding, X., and Tang, J. (2017). Promising therapeutic role of miR-27b in tumor. Tumour Biol 39, 101042831769165.

Doyle, L.P., and Hutchings, L.M. (1946). A transmissible gastroenteritis in pigs. J Am Vet Med Assoc 108, 257-259.

Du, H., Cui, S., Li, Y., Yang, G., Wang, P., Fikrig, E., and You, F. (2018). miR-221 negatively regulates innate anti-viral response. PLoS ONE 13, e0200385.

Fehr, A.R., and Perlman, S. (2015). Coronaviruses: an overview of their replication and pathogenesis. Methods Mol Biol 1282, 1-23.

Fink, S.L., Jayewickreme, T.R., Molony, R.D., Iwawaki, T., Landis, C.S., Lindenbach, B.D., and Iwasaki, A. (2017). IRE1 $\alpha$ promotes viral infection by conferring resistance to apoptosis. Sci Signal 10, eaai7814.

Forster, S.C., Tate, M.D., and Hertzog, P.J. (2015). MicroRNA as type I interferon-regulated transcripts and modulators of the innate immune response. Front Immunol 6, 334.

Fung, T.S., Huang, M., and Liu, D.X. (2014). Coronavirus-induced ER stress response and its involvement in regulation of coronavirus-host interactions. Virus Res 194, 110-123.

Fung, T.S., and Liu, D.X. (2014). Coronavirus infection, ER stress, apoptosis and innate immunity. Front Microbiol 5, 296.

Gatto, G., Rossi, A., Rossi, D., Kroening, S., Bonatti, S., and Mallardo, M. (2008). Epstein-Barr virus latent membrane protein 1 trans-activates miR-155 transcription through the NF- $\mathrm{kB}$ pathway. Nucleic Acids Res $36,6608-6619$.

Hassan, I.H., Zhang, M.S., Powers, L.S., Shao, J.Q., Baltrusaitis, J., Rutkowski, D.T., Legge, K., and Monick, M.M. (2012). Influenza A viral replication is blocked by inhibition of the inositol-requiring enzyme 1 (IRE1) stress pathway. J Biol Chem 287, 4679-4689.

Hassler, J., Cao, S.S., and Kaufman, R.J. (2012). IRE1, a double-edged sword in pre-miRNA slicing and cell death. Dev Cell 23, 921-923.

Heindryckx, F., Binet, F., Ponticos, M., Rombouts, K., Lau, J., Kreuger, J., and Gerwins, P. (2016). Endoplasmic reticulum stress enhances fibrosis through IRE1 $\alpha$-mediated degradation of miR-150 and XBP-1 splicing. EMBO Mol Med 8, 729-744.

Huang, J., Wang, F., Argyris, E., Chen, K., Liang, Z., Tian, H., Huang, W., Squires, K., Verlinghieri, G., and Zhang, H. (2007). Cellular microRNAs contribute to HIV-1 latency in resting primary $\mathrm{CD}^{+} \mathrm{T}$ lymphocytes. Nat Med 13, 1241-1247.

Kai, Z.S., and Pasquinelli, A.E. (2010). MicroRNA assassins: factors that regulate the disappearance of miRNAs. Nat Struct Mol Biol 17, 5-10.

Lecellier, C.H., Dunoyer, P., Arar, K., Lehmann-Che, J., Eyquem, S., Himber, C., Saib, A., and Voinnet, O. (2005). A cellular microRNA mediates antiviral defense in human cells. Science 308, 557-560.

Lee, A.H., Iwakoshi, N.N., and Glimcher, L.H. (2003). XBP-1 regulates a subset of endoplasmic reticulum resident chaperone genes in the unfolded protein response. Mol Cell Biol 23, 7448-7459.

Ma, Y., Wang, C., Xue, M., Fu, F., Zhang, X., Li, L., Yin, L., Xu, W., Feng, L., and Liu, P. (2018). The coronavirus transmissible gastroenteritis virus evades the type I interferon response through IRE1alpha-mediated manipulation of the microRNA miR-30a-5p/SOCS1/3 axis. J Virol 92.

Mahajan, V.S., Drake, A., and Chen, J. (2009). Virus-specific host miRNAs: antiviral defenses or promoters of persistent infection? Trends Immunol 30, 1-7.

Mallick, B., Ghosh, Z., and Chakrabarti, J. (2009). MicroRNome analysis unravels the molecular basis of SARS infection in bronchoalveolar stem cells. PLoS ONE 4, e7837.

O'Connell, R.M., Taganov, K.D., Boldin, M.P., Cheng, G., and Baltimore, D. (2007). MicroRNA-155 is induced during the macrophage inflammatory response. Proc Natl Acad Sci USA 104, 1604-1609.

O’Neill, L.A., Sheedy, F.J., and McCoy, C.E. (2011). MicroRNAs: the finetuners of Toll-like receptor signalling. Nat Rev Immunol 11, 163-175.

Peng, X., Gralinski, L., Ferris, M.T., Frieman, M.B., Thomas, M.J., Proll, S., Korth, M.J., Tisoncik, J.R., Heise, M., Luo, S., et al. (2011). Integrative deep sequencing of the mouse lung transcriptome reveals 
differential expression of diverse classes of small RNAs in response to respiratory virus infection. mBio 2 .

Potenza, N., Papa, U., Mosca, N., Zerbini, F., Nobile, V., and Russo, A. (2011). Human microRNA hsa-miR-125a-5p interferes with expression of hepatitis B virus surface antigen. Nucleic Acids Res 39, 5157-5163.

Pritchard, G. (1987). Transmissible gastroenteritis in endemically infected breeding herds of pigs in East Anglia, 1981-85. Vet Record 120, 226230.

Rivera-Ramírez, E., Méndez, L.B., Ortíz-Rivera, A., Rodríguez-Cotto, R.I., and Jiménez-Vélez, B. (2019). A novel role of PM extracts on the posttranscriptional control of pro-inflammatory mediators, IL-6 and CXCL8. Atmosphere 10, 270.

van Rooij, E., and Olson, E.N. (2007). MicroRNAs: powerful new regulators of heart disease and provocative therapeutic targets. J Clin Invest 117, 2369-2376.

Xue, M., Fu, F., Ma, Y., Zhang, X., Li, L., Feng, L., and Liu, P. (2018). The PERK arm of the unfolded protein response negatively regulates transmissible gastroenteritis virus replication by suppressing protein translation and promoting type I interferon production. J Virol 92.

Yu, Z., and Hecht, N.B. (2008). The DNA/RNA-binding protein, translin, binds microRNA122a and increases its in vivo stability. J Androl 29, 572-579.

Zhang, K., Liu, H., Song, Z., Jiang, Y., Kim, H., Samavati, L., Nguyen, H. M., and Yang, Z.Q. (2020). The UPR transducer IRE1 promotes breast cancer malignancy by degrading tumor suppressor microRNAs. iScience 23, 101503.

Zhao, X., Song, X., Bai, X., Fei, N., Huang, Y., Zhao, Z., Du, Q., Zhang, H., Zhang, L., and Tong, D. (2016). miR-27b attenuates apoptosis induced by transmissible gastroenteritis virus (TGEV) infection via targeting runt-related transcription factor 1 (RUNX1). PeerJ 4, e1635.

Zheng, H., Xu, L., Liu, Y., Li, C., Zhang, L., Wang, T., Zhao, D., Xu, X., and Zhang, Y. (2018). MicroRNA-221-5p inhibits porcine epidemic diarrhea virus replication by targeting genomic viral $\mathrm{RNa}$ and activating the NF- $\kappa$ B pathway. Int J Mol Sci 19, 3381.

\section{SUPPORTING INFORMATION}

The supporting information is available online at https://doi.org/10.1007/s11427-021-1967-x. The supporting materials are published as submitted, without typesetting or editing. The responsibility for scientific accuracy and content remains entirely with the authors. 